\title{
Quantification of the impact of large and small scale instabilities on the fast-ion confinement in ASDEX Upgrade
}

\author{
B. Geiger,${ }^{1}$ M. Weiland, ${ }^{1}$ A. Mlynek,${ }^{1}$ M. Reich,${ }^{1}$ A. Bock,${ }^{1}$ M. Dunne, ${ }^{1}$ R. Dux,${ }^{1}$ E. Fable,${ }^{1}$ R. Fischer,${ }^{1}$ \\ M. Garcia-Munoz, ${ }^{2}$ J. Hobirk, ${ }^{1}$ C. Hopf,${ }^{1}$ S. Nielsen, ${ }^{3}$ T. Odstrcil,${ }^{1}$ C. Rapson, ${ }^{1}$ D. Rittich, ${ }^{1}$ F. Ryter, ${ }^{1}$ \\ M. Salewski, ${ }^{3}$ P. A. Schneider,${ }^{1}$ G. Tardini, ${ }^{1}$ M. Willensdorfer ${ }^{1}$ and the ASDEX Upgrade Team $^{1}$ \\ ${ }^{1}$ Max Planck Institute for Plasma Physics, Boltzmannstr. 2, 85748 Garching, Germany \\ ${ }^{2}$ Faculty of Physics, University of Seville, Spain \\ ${ }^{3}$ Technical University of Denmark, Department of Physics, Dk-2800 Kgs. Lyngby, Denmark
}

(Dated: September 17, 2014)

\begin{abstract}
The confinement of neutral beam (NBI) generated fast ions has been investigated at the ASDEX Upgrade tokamak. In plasmas that exhibit strong sawtooth crashes, significant sawtooth-induced internal redistribution of mainly passing fast ions is observed which is in very good agreement with theoretical predictions based on the Kadomtsev model. Between the sawtooth crashes, fishbone modes are excited which, however, do not cause measurable changes of the global fast-ion population. During experiments with on- and off-axis NBI and without strong magneto hydrodynamic (MHD) modes, the fast-ion measurements agree very well with neo-classical predictions. This shows that the MHD-induced (large scale), as well as a possible turbulence-induced (small scale) fast-ion transport is negligible under these conditions. However, in discharges performed to study the off-axis NBI current drive efficiency with up to $10 \mathrm{MW}$ of heating power, the fast-ion measurements agree best with theoretical predictions that assume a weak level anomalous fast-ion transport. This is also in agreement with measurements of the internal inductance, a Motional Stark Effect diagnostic and a novel polarimetry diagnostic: The fast-ion driven current profile is clearly modified when changing the NBI injection geometry and the measurements agree best with predictions that assume weak anomalous fast-ion diffusion.
\end{abstract}

PACS numbers: 52.55.Fa, 52.50.Gj, 52.55.Tn, 52.55.Wq

\section{INTRODUCTION}

The confinement of fast ions is an important topic in fusion research because these suprathermal particles are responsible for plasma heating [1] and current drive [2]. In present day fusion devices, fast ions are generated by neutral beam injection (NBI) or ion cyclotron heating (ICRH) and their slowing down distribution in unperturbed plasmas can be described by collisions with electrons and ions (neo-classical). In the presence of magneto-hydrodynamic (MHD) modes and turbulence, the fast-particle confinement can be affected by additional anomalous fast-ion redistribution. The physics background of this anomalous transport must be investigated in view of future fusion devices that will exhibit large fast-ion populations: Strong MHD modes and turbulence may not only limit the heating and current drive performance but could also damage the device due to the redistribution of the fast ions onto the first wall [3].

Significant anomalous transport of fast ions has been observed due to sawtooth crashes and fishbones. Both are two prominent MHD instabilities that often occur in tokamak plasmas. They are linked to the growth of a $\mathrm{n}=1, \mathrm{~m}=1$ kink mode [4] at the $\mathrm{q}=1$ surface (here, $\mathrm{n}$ and $\mathrm{m}$ are the toroidal and poloidal mode numbers, respectively and $\mathrm{q}$ is the safety factor) and appear periodically. While the fishbone instability mainly interacts with trapped fast ions (it is resonant with the precession motion of the banana-tips of trapped particles), sawtooth crashes cause a fast magnetic reconnection in the plasma center and, hence, redistribute heat, particles and momentum. Already 20 years ago, a clear reduction of measured neutron rates was observed at the JET [5] and TFTR [6] tokamaks during sawtooth crashes, indicating a significant fast-ion transport. In recent years, measurements at DIII-D [7] and TEXTOR [8] showed that sawteeth redistribute passing fast ions more strongly than trapped ions (as predicted by theory [9] above a given critical fast-ion energy) and measurements at MAST [10] and JET [11] evidenced a reduction of the density of confined fast ions in the presence of fishbones.

The effect of small scale turbulence on the fast ions is, to a first approximation, expected to be negligible because the turbulent fields are supposed to average out over the fast ions' large gyro orbits and trajectories that deviate from the flux surfaces [12]. However, gyro-kinetic simulations show that the turbulence-induced variations of the magnetic field can hamper this orbit averaging mechanism [13] and hence, yield anomalous diffusion also for the suprathermal 

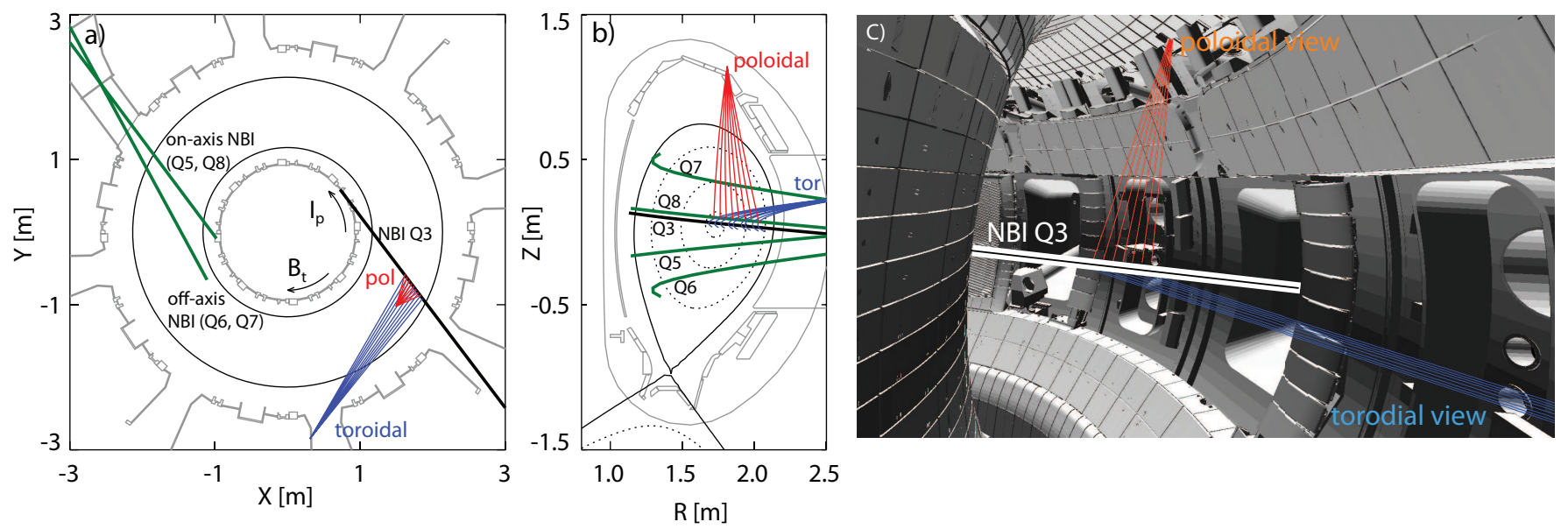

FIG. 1: Top-down (a) poloidal (b) and 3D (c) view on the AUG tokamak. The neutral beams with $93 \mathrm{keV}$ maximum injection energy are plotted in green and NBI Q3 $(60 \mathrm{keV})$ which is used as diagnostic beam for the FIDA diagnostic is given in black. The toroidal LOS of the FIDA diagnostic are indicated in blue and the poloidal ones are displayed in red.

particles. Experimentally, differences between measurements and neo-classical expectations were observed at DIIID [14]. Furthermore, the measurements from a Motional Stark Effect (MSE) diagnostic [15] at ASDEX Upgrade (AUG) were almost identical for on-axis and off-axis NBI heating which cannot be explained by neoclassical fast-ion current drive [16]. Turbulence-induced anomalous fast-ion transport was therefore attributed to these observations as the analyzed discharges were MHD-quiescent, i.e. no significant mode activity was observed using soft X-ray and magnetic measurements. This is, however, in contradiction to more recent measurements of the fast-ion slowing down distribution that do not show a clear impact of turbulence [17-19].

Over the last few years, AUG has been equipped with a set of new fast-ion diagnostics. In particular a fast-ion D-alpha (FIDA[20]) spectroscopy diagnostic has been put into operation [21] that is absolutely calibrated and, hence, allows us to compare predicted fast-ion densities with the experimental data. Moreover, a revised MSE diagnostic and a novel DCN polarimeter [22] permit us to investigate the effect of the fast ions on the current profile. The effects of large and small scale instabilities on the fast-ion confinement and on the fast-ion current drive efficiency can, consequently, be studied and are presented here.

This paper is organized as follows: First, the experimental setup at AUG is presented focusing, on the FIDA diagnostic. Then, experiments using NBI heating are presented that show strong sawtooth crashes and fishbone activity. A comparison is given between the experimental data and theoretical predictions from TRANSP [23] that include the Kadomtsev model [24]. In the fourth section, investigations of the fast-ion confinement in MHD-quiescent discharges with $5 \mathrm{MW}$ of on- and off-axis NBI are presented. Section five is devoted to the analysis of a discharge with up to $10 \mathrm{MW}$ of heating power that was preformed to study the off-axis NBI current drive efficiency. The measured radial fast-ion profiles are compared with simulations and changes of the current profile due to the application of off-axis NBI are studied. Finally, a short conclusion and summary are given.

\section{EXPERIMENTAL SETUP}

ASDEX Upgrade [25] is a medium size tokamak with minor and major radii of $0.5 \mathrm{~m}$ and $1.65 \mathrm{~m}$, respectively resulting in an aspect ratio similar to ITER [26]. The experiments presented here were performed in deuterium plasmas with a central toroidal magnetic field strength of about $-2.5 \mathrm{~T}$ and a feedback controlled plasma current at either $800 \mathrm{kA}$ or $1 \mathrm{MA}$. Five different neutral beams, delivering each $2.5 \mathrm{MW}$ of heating power were used for this investigation. The geometry of these NBIs, injecting fast deuterium neutrals in the plasma current direction, is described in figure $1 \mathrm{a}$ and $1 \mathrm{~b}$ on a top-down and poloidal view. As can be seen in the poloidal view, three of the beam sources inject radially into the plasma center whereas two beams, i.e. Q6 and Q7, have an off-axis and tangential geometry and are used for off-axis current drive experiments. Simulated (TRANSP) deposition positions of the injected neutrals from these NBIs, as well as the corresponding fast-ion densities, are plotted in figure 2 for 

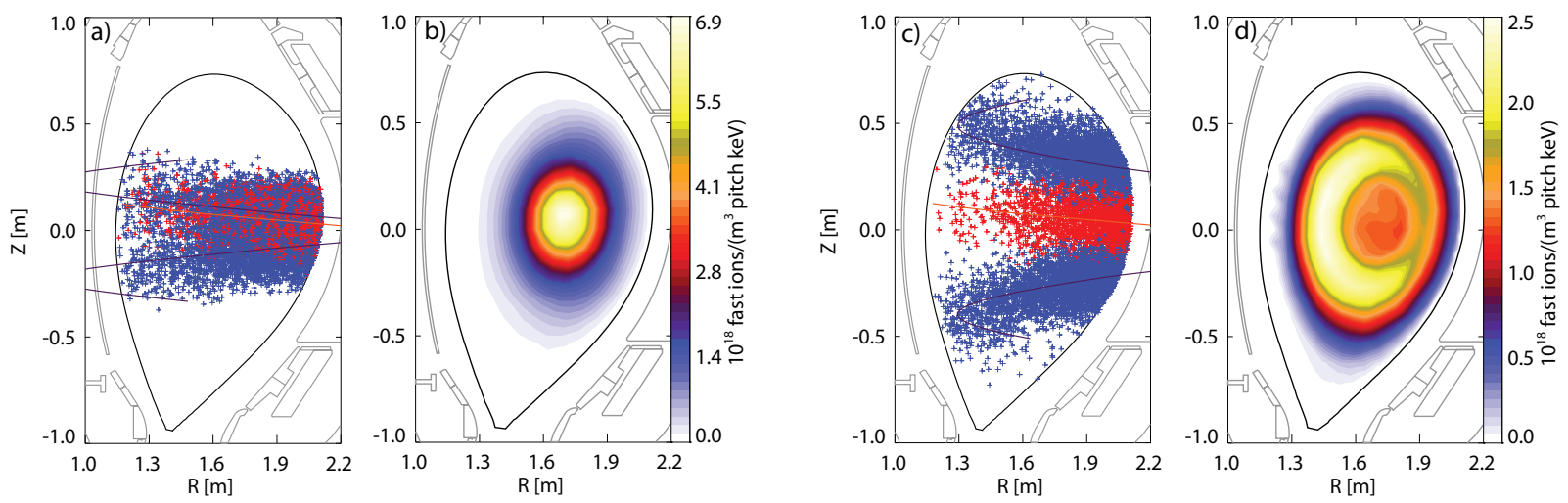

FIG. 2: Simulated fast-ion deposition positions (of Monte Carlo markers in TRANSP) and corresponding fast-ion densities (integrated over all pitch angles and energies above $30 \mathrm{keV}$ ) on a poloidal view for discharge \#30840. Figures a and b represent the fast ions from NBI sources Q3, Q5 and Q8 (\#30840 at 3.9s). Figures c and d represent the fast ions from NBI sources Q3, Q6 and Q7 (\#30840 at 5.0 s). The deposition positions calculated for the $93 \mathrm{keV}$ beams are given in blue. The simulated deposition positions of NBI Q3 $(60 \mathrm{keV})$ are plotted in red.
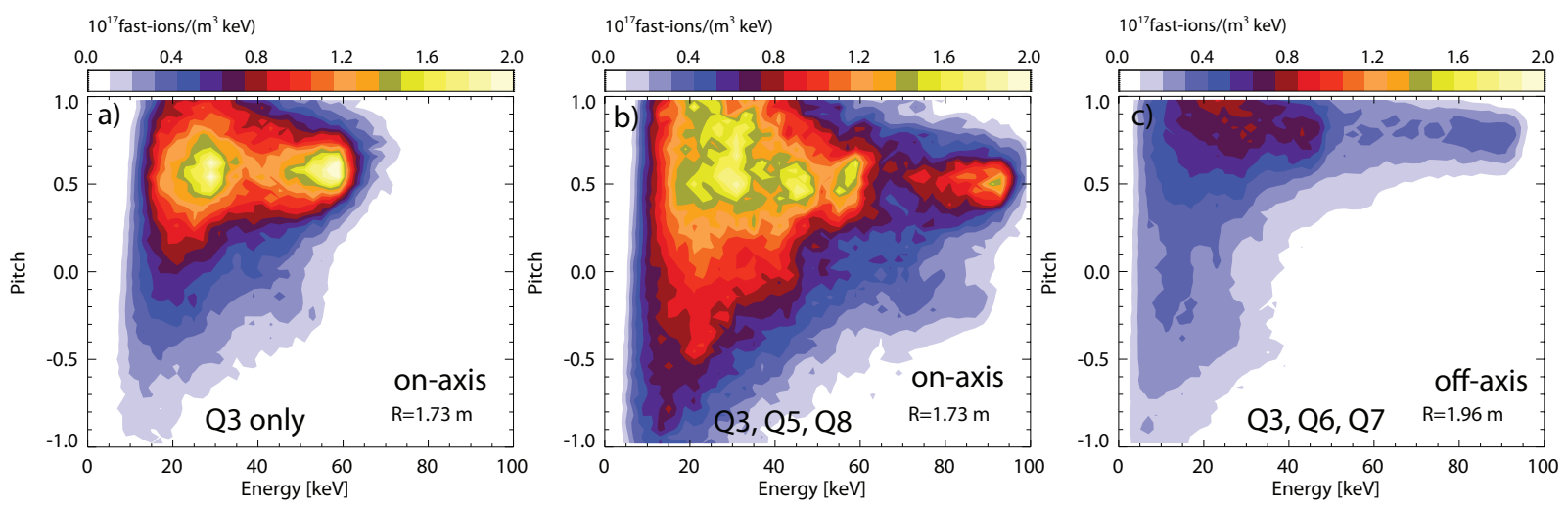

FIG. 3: Velocity-space distribution of fast ions as predicted by TRANSP. a) Central fast-ion velocity-space distribution during operation of NBI Q3 in discharge \#30809 at 2.5 s. b) Central velocity-space distribution when sources Q3, Q5 and Q8 are on in \#30840 at 3.9 s. c) Off-axis velocity-space distribution when sources Q3, Q6 and Q7 are on in \#30840 at 5.0 s

two representative cases. Figures $2 \mathrm{a}$ and $2 \mathrm{~b}$ represent the fast-ion deposition and density profiles during operation of the three on-axis NBI sources Q3, Q5 and Q8. Figure 2c and 2d show the deposition and density profiles for the operation of Q3 together with the two off-axis sources (Q6 and Q7). Representative velocity-space distributions for the two cases are depicted in figure $3 \mathrm{~b}$ and $3 \mathrm{c}$ as a function of energy and pitch for two radial positions $\left(\right.$ pitch $=v_{\|} / v$ where $v$ is the absolute value of the fast-ion velocity and $v_{\|}$is its component anti-parallel to the magnetic field). In addition, figure 3a shows the central velocity-space distribution of fast ions from just NBI Q3. Most of the fast ions have positive pitch values, corresponding to the co-current neutral beam injection geometry. In figure 3a, two peaks can be observed in the fast-ion velocity space distribution at pitch values close to 0.6. These can be attributed to the full injection energy of NBI Q3 of $60 \mathrm{keV}$ and to the injection of half $(30 \mathrm{keV})$ and one third $(20 \mathrm{keV})$ energetic particles which is characteristic for positive ion sources in NBI systems. The maximum energy in figures $3 \mathrm{~b}$ and $3 \mathrm{c}$ corresponds to the full injection energy of $93 \mathrm{keV}$ of sources Q5-Q8. The pitch values of the fast ions from sources Q5 and Q8 are similar to those of fast ions injected by Q3 since the geometry of the sources is comparable. In contrast, the paths of the off-axis sources are close to tangential to the magnetic field lines. Therefore, these neutral beams generate fast ions with pitch values close to 1 (see figure $3 \mathrm{c}$ ).

The main diagnostic used for this investigation is a fast-ion D-alpha (FIDA) spectroscopy diagnostic. This diagnostic utilizes the principle of charge exchange recombination spectroscopy (CXRS [27]) and is well suited for measuring fast ions present in the velocity-space region populated by the individual NBIs. Strongly Doppler shifted Balmer alpha radiation $(\mathrm{n}=3 \rightarrow \mathrm{n}=2,656.1 \mathrm{~nm})$ is analyzed that is emitted by fast ions after receiving an electron along the path 

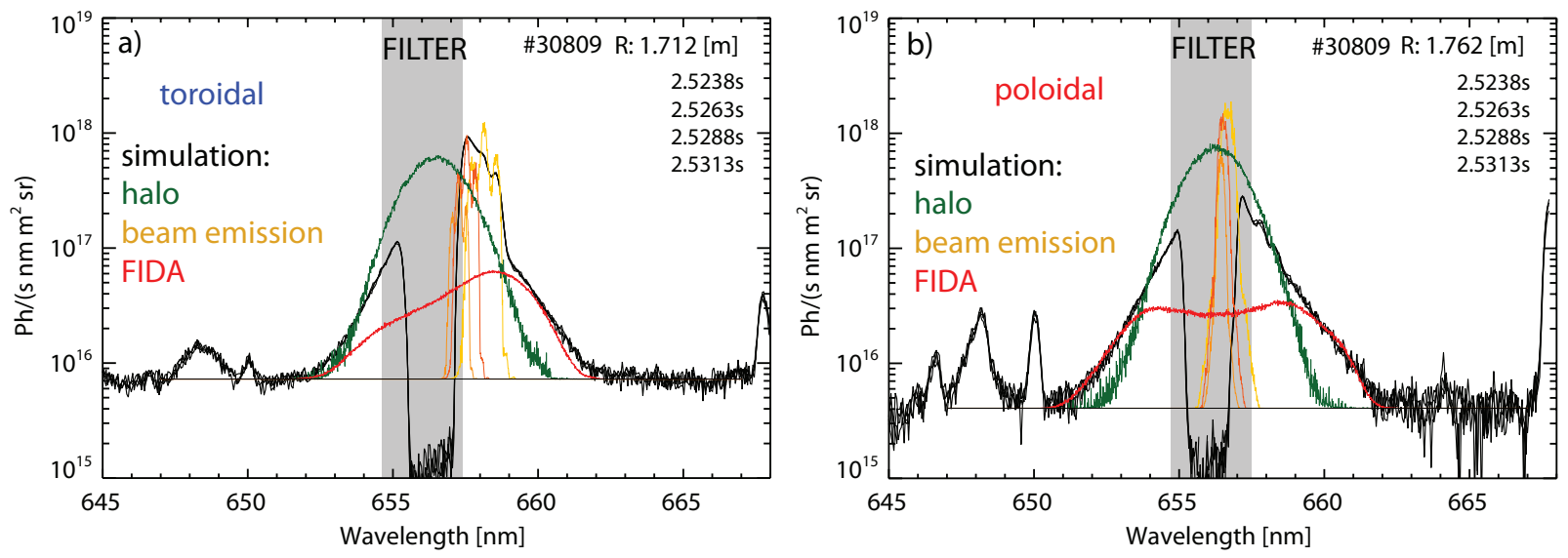

FIG. 4: Spectra from a poloidal and a toroidal line of sight of the FIDA diagnostic measured before a sawtooth crash in discharge \#30809 (note the semi logarithmic scale). In addition, simulated spectra from FIDASIM are plotted. The simulated halo radiation is displayed in green color, the beam emission in yellow/orange and the simulated FIDA radiation, based on a theoretical fast-ion distribution function from TRANSP, is plotted in red.

of neutral beam Q3. By using several lines of sight (LOS) that intersect this NBI path, good radial coverage of the plasma is obtained. The LOS geometry of the FIDA diagnostic is plotted in figure 1 with toroidal views illustrated in blue and poloidal views shown in red. Example spectra from a central toroidal LOS and a central poloidal LOS are shown in figure 4 for four successive time points during operation of NBI Q3 only (actually measured in discharge \#30809 which will be discussed in detail in section III.). Between $655 \mathrm{~nm}$ and $657 \mathrm{~nm}$, the very intense D-alpha radiation from the plasma edge is blocked by a filter (actually a wire in the FIDA spectrometer close to the camera of the diagnostic) because it would cause saturation of the diagnostic's CCD camera. The experimental data in figure 4 is compared with simulated spectra from FIDASIM [28]. FIDASIM is as a Monte Carlo code on a 3D simulation grid that calculates synthetic FIDA spectra based on a given theoretical fast-ion distribution function. The simulation plotted in red in figure 4 shows the FIDA radiation that corresponds to a neo-classical fast-ion distribution function from TRANSP [23]. The FIDA radiation can only be analyzed at strongly red and blue-shifted wavelengths because at lower wavelength shifts, it is obscured by other spectral components. The strongest contributions, in addition to the FIDA radiation, are the beam emission from the injected neutrals (the corresponding prediction from FIDASIM is plotted in yellow/orange) and halo emission (simulation shown in green) that is charge exchange radiation from thermal deuterium ions. Moreover, passive components (also present without NBI Q3) limit the observable wavelength range. In particular, impurity line radiation of Oxygen (OV at $650.02 \mathrm{~nm}$ ) limits the observation of blue-shifted FIDA radiation.

For the investigation presented here, FIDA radiation is analyzed between $651 \mathrm{~nm}$ and $652 \mathrm{~nm}$ and between $660 \mathrm{~nm}$ and $661 \mathrm{~nm}$. The Bremsstrahlung radiation, additionally present in these wavelength intervals, is modeled as a constant that is independent of the wavelength. The region in the velocity space that is accessed when analyzing the FIDA radiation in a given wavelength range depends on the LOS geometry, i.e. on the projection angle between the magnetic field and the LOS. Hence, different regions in velocity space are monitored by the toroidal $\left(\sim 10^{\circ}\right)$ and poloidal LOS $\left(\sim 70^{\circ}\right)$. The velocity-space regions, addressed when analyzing red and blue-shifted FIDA radiation from the two viewing geometries, are illustrated in figures $5 \mathrm{a}-5 \mathrm{~d}$ in terms of weight functions $[29,30]$. As can be seen in figure 5a, the toroidal LOS on the blue-shifted side of the spectra can only detect counter rotating passing fast ions above $30 \mathrm{keV}$ with pitches close to -1 . In contrast, the toroidal LOS on the red-shifted side (figure $5 \mathrm{~b}$ ) are sensitive to co-rotating fast ions. The poloidal LOS cover a broad range in velocity space. The blue-shifted part of the spectra is more sensitive to negative pitch values (see figure $5 \mathrm{c}$ ) and the red-shifted part measures fast ions with pitch values biased towards the positive side (figure $5 \mathrm{~d}$ ). It should be noted that, based on these weight functions, a tomographic reconstruction of the fast-ion velocity space distribution can be performed [31].

Due to the injection geometry of the NBI sources at AUG, the fast-ion distribution functions have mainly positive pitch angles (see figure 3). Fast ions with negative pitch values are only produced by pitch angle scattering (which becomes significant at fast-ion energies below $18.6 \cdot T_{e}[1]$ ) and by trapped orbits. Therefore, the overlap of the weight functions with the distribution functions is small for blue-shifted wavelengths and large for red-shifted wavelengths. 

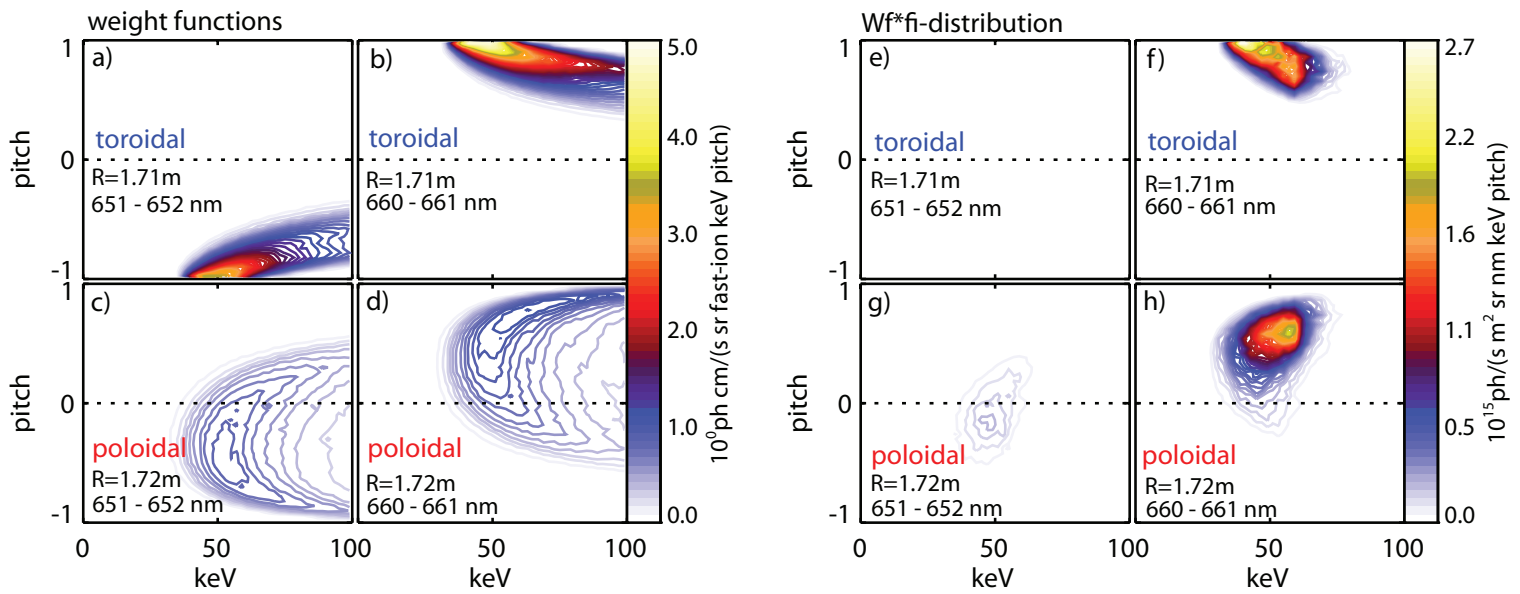

FIG. 5: a-d) Weight functions for two LOS of the FIDA diagnostic for wavelengths between $651 \mathrm{~nm}$ and $652 \mathrm{~nm}$ as well as for wavelengths between $660 \mathrm{~nm}$ and $661 \mathrm{~nm}$. The viewing angle of the toroidal view in the upper panels is $\sim 10^{\circ}$, and that of the poloidal view in the lower panel is $\sim 70^{\circ}$ relative to the magnetic field. e-h) Product of the velocity space distribution shown in figure $3 \mathrm{a}$ and of the weight functions.

Figures 5e-5h show the product of the weight functions with the representative velocity-space distribution of fast ions generated by NBI Q3, shown in figure 3a. The significant overlap of the toroidal weight function for red-shifted wavelengths with the fast-ion distribution function (see figure 5f) explains the asymmetric shape of the FIDA radiation in the toroidal spectrum (4a): the strongest FIDA contribution is visible at red-shifted wavelengths. Interestingly, the poloidal LOS allows us to measure FIDA radiation as well between $651 \mathrm{~nm}$ and $652 \mathrm{~nm}$ (see figure 4b). This radiation corresponds to fast particles with pitch values in the vicinity of zero. When analyzing this radiation we, hence, obtain information on mainly trapped fast particles.

\section{SAWTOOTH AND FISHBONE EXPERIMENTS}

\begin{tabular}{|l|l|l|l|l|l|}
\hline $\mathrm{B}_{\mathrm{t}}$ & $\mathrm{I}_{\mathrm{p}}$ & $\mathrm{n}_{\mathrm{e}}$ & $\mathrm{q} 95$ & $\rho_{\mathrm{t}}(\mathrm{q}=1)$ & $\delta \mathrm{B}_{\max }$ \\
\hline$-2.4 \mathrm{~T}$ & $1.0 \mathrm{MA}$ & $3.9 \times 10^{19} \mathrm{~m}^{-3}$ & 4.09 & 0.30 & $3 \mathrm{mT}$ \\
\hline
\end{tabular}

TABLE I: Main discharge parameters of \#30809 consisting of the toroidal magnetic field, $B_{t}$, the plasma current, $I_{p}$, the average electron density, $\mathrm{n}_{\mathrm{e}}$, the safety factor at $95 \%$ of magnetic flux, $\mathrm{q} 95$, the position of the $\mathrm{q}=1$ surface, $\rho_{\mathrm{t}}(\mathrm{q}=1)$ and the approximate maximum magnetic perturbation amplitude induced by $\mathrm{n}=1, \mathrm{~m}=1$ modes, $\delta B_{\max }$.

Discharges with 2.5 MW of heating power from NBI source Q3 have been conducted with 1 MA of current and $-2.4 \mathrm{~T}$ of magnetic field (see table I). Figures $6 \mathrm{a}, 6 \mathrm{~b}$ and $6 \mathrm{c}$ display characteristic time traces, a spectrogram and the approximate magnetic perturbation amplitude induced by $n=1, m=1$ modes for a representative discharge. The approximate magnetic perturbation amplitude has been calculated based on measurements of a magnetic pick-up coil at $\mathrm{R}=2.25 \mathrm{~m}$ by assuming that the amplitude decays with $\frac{1}{r^{m+1}}$. The sawtooth crashes become visible by spikes in the magnetic perturbation amplitude and by the drops of the central electron density and temperature, the central ion temperature and plasma rotation (see figure 6a). This behavior is well known and expected as the sawtooth instability is linked to the magnetic reconnection of field lines in the plasma center and redistributes heat, particles and momentum.

The relatively low electron densities and high temperatures in discharge \#30809 yield low collision frequencies $\left(\sim n_{\mathrm{e}} / T_{e}^{3 / 2}\right)$, long slowing down times and consequently a significant fast-ion fraction in the plasma. Hence, the FIDA diagnostic measures with good signal to noise ratio and a clear fast-ion redistribution due to the sawtooth crashes can be observed. As an example, figure $7 \mathrm{a}$ and $7 \mathrm{~b}$ show spectra from a central toroidal LOS and a central poloidal LOS, respectively. The data plotted in black corresponds to four time points before the sawtooth crash at about $2.5 \mathrm{~s}$ in discharge \#30809. The spectra in red represent the measurements after that sawtooth crash. As can 

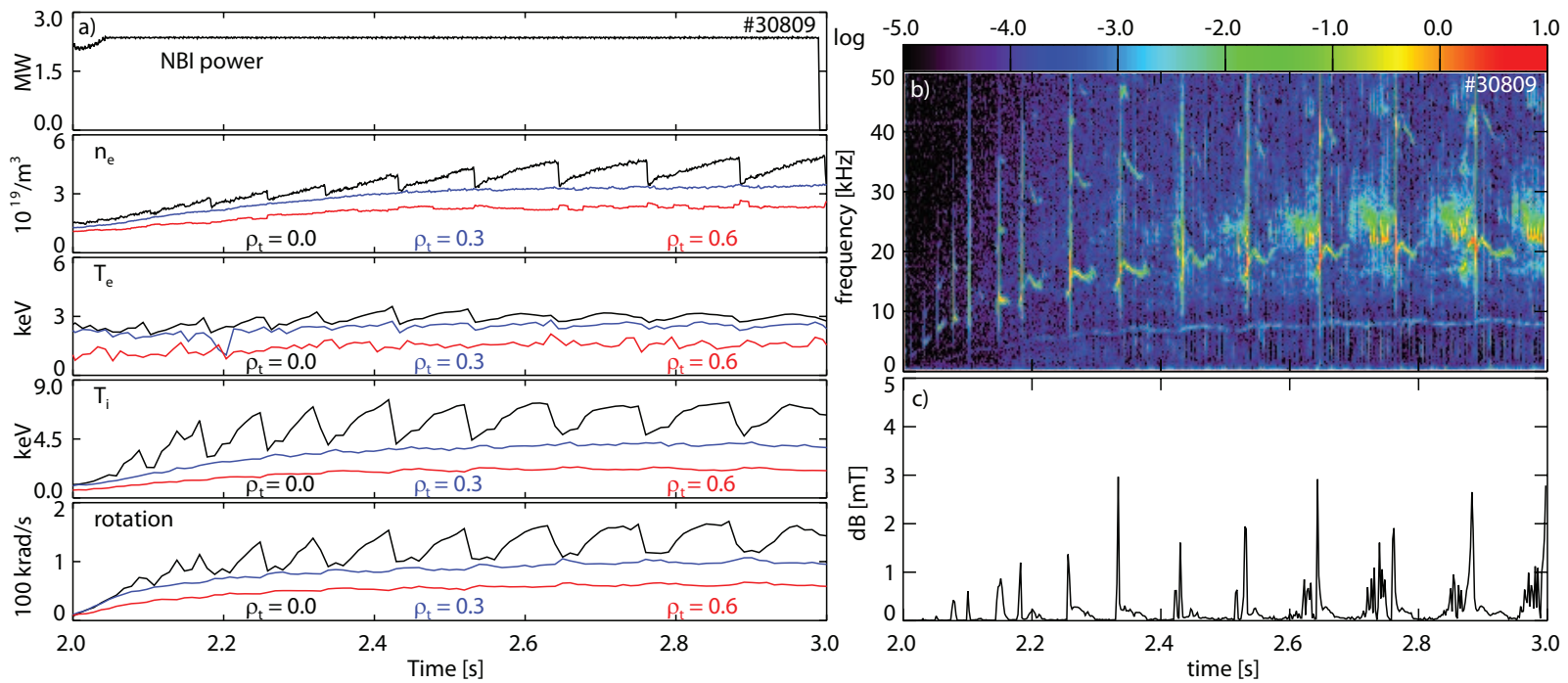

FIG. 6: a) Characteristic time traces of discharge \#30809 showing the NBI heating power, the electron density and temperature, the ion temperature and the toroidal plasma rotation. b) Magnetic spectrogram of \#30809 showing sawtooth crashes that appear at all frequencies and fishbone instabilities (before the sawteeth from $2.5 \mathrm{~s}$ on). c) Approximate magnetic perturbation amplitude of $\mathrm{n}=1, \mathrm{~m}=1$ modes based on measurements of a magnetic pick-up coil.
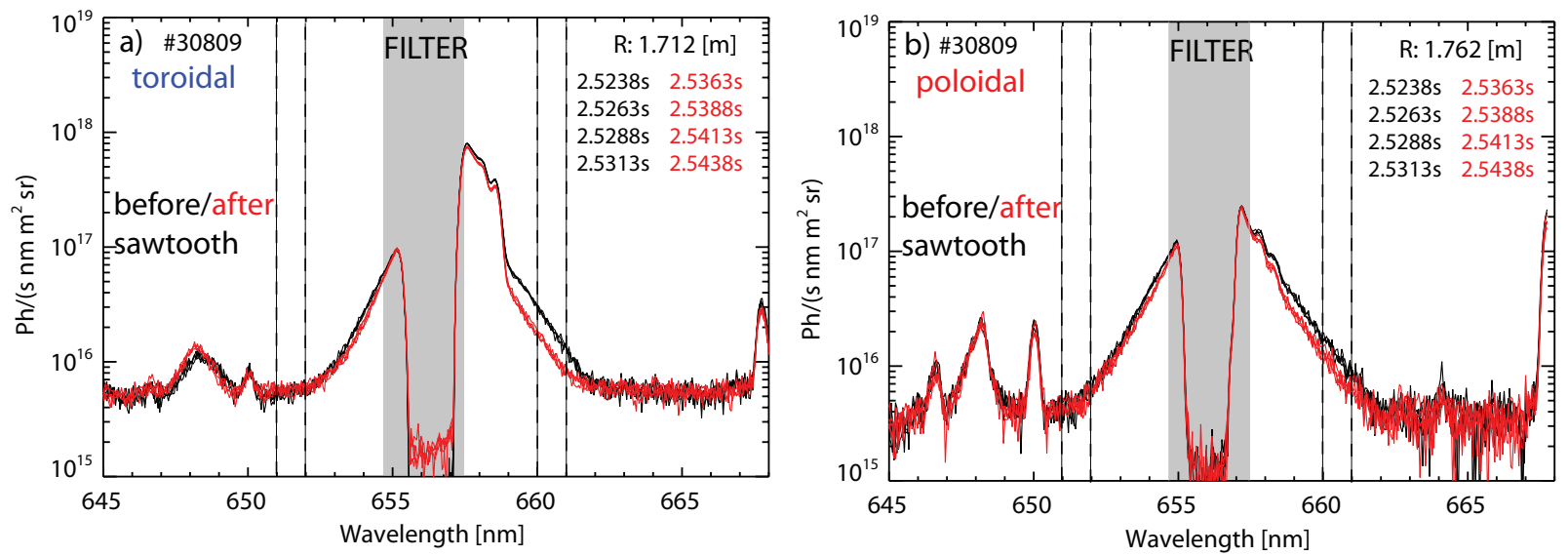

FIG. 7: FIDA spectra from a central toroidal LOS (a) and a central poloidal LOS (b). In black, four successive spectra are shown, measured before a sawtooth crash. The data in red corresponds to four time points after a sawtooth crash. The dashed lines illustrate the two wavelength-regions that are used when analyzing the FIDA radiation (blue and red-shifted FIDA radiation).

be seen, the FIDA radiation observed with the toroidal LOS shows a very clear change at red-shifted wavelengths while the FIDA radiation measured with the poloidal LOS shows a weaker effect of the sawtooth crashes. Radial profiles of the FIDA radiation, measured with the toroidal and poloidal LOS, are shown in figures 8a and 8b as a function of the normalized toroidal flux coordinate, $\rho_{\mathrm{t}}$. The error-bars of the measurement correspond to the readout and photon noise of the FIDA diagnostic. The profiles were calculated by integrating the FIDA radiation per channel between $660 \mathrm{~nm}$ and $661 \mathrm{~nm}$ and by subtracting the level of Bremsstrahlung that is modeled by a flat line. In addition, constant offsets have been subtracted from the toroidal $\left(0.2 \cdot 10^{16} \mathrm{ph} /\left(\mathrm{s} \mathrm{sr} \mathrm{m} \mathrm{m}^{2}\right)\right)$ and poloidal $\left(\left(0.1 \cdot 10^{16} \mathrm{ph} /\left(\mathrm{s} \mathrm{sr} \mathrm{m}^{2}\right)\right)\right.$ profiles in order to match the simulation which is shown by solid lines. These offsets can be explained by passive FIDA radiation that is caused by charge exchange reactions between fast ions and neutrals at the plasma edge and which can be roughly described by a constant in the radial profiles (see [21]).

The simulated profiles from FIDASIM represent a fast-ion distribution function from TRANSP which has been supplied with time-dependent information on the plasma current and separatrix position, on the geometry, power and 

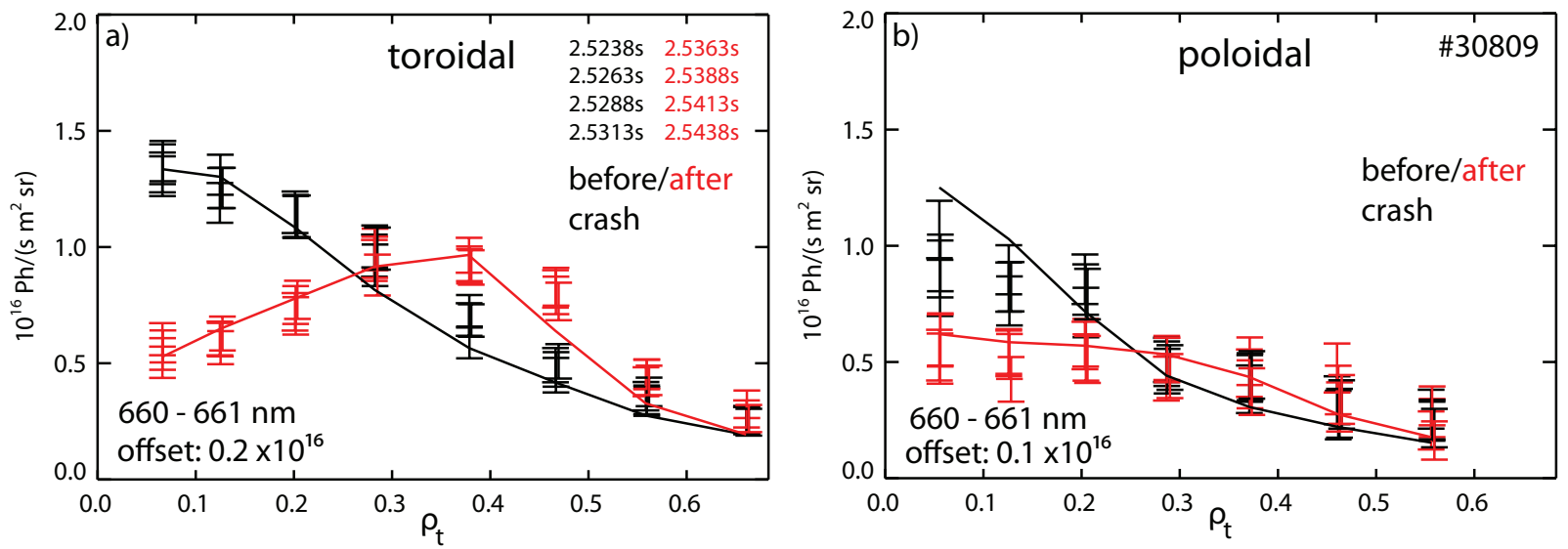

FIG. 8: Red-shifted radial FIDA intensity profiles acquired by the toroidal (a) and poloidal (b) LOS. The profiles were measured before (black) and after (red) a sawtooth crash in discharge \#30809 at $2.532 \mathrm{~s}$. The error bars correspond to the readout and photon noise contained in the FIDA spectra. The solid lines show synthetic FIDA intensity profiles from FIDASIM that are based on the TRANSP predictions, plotted in figure 9b.
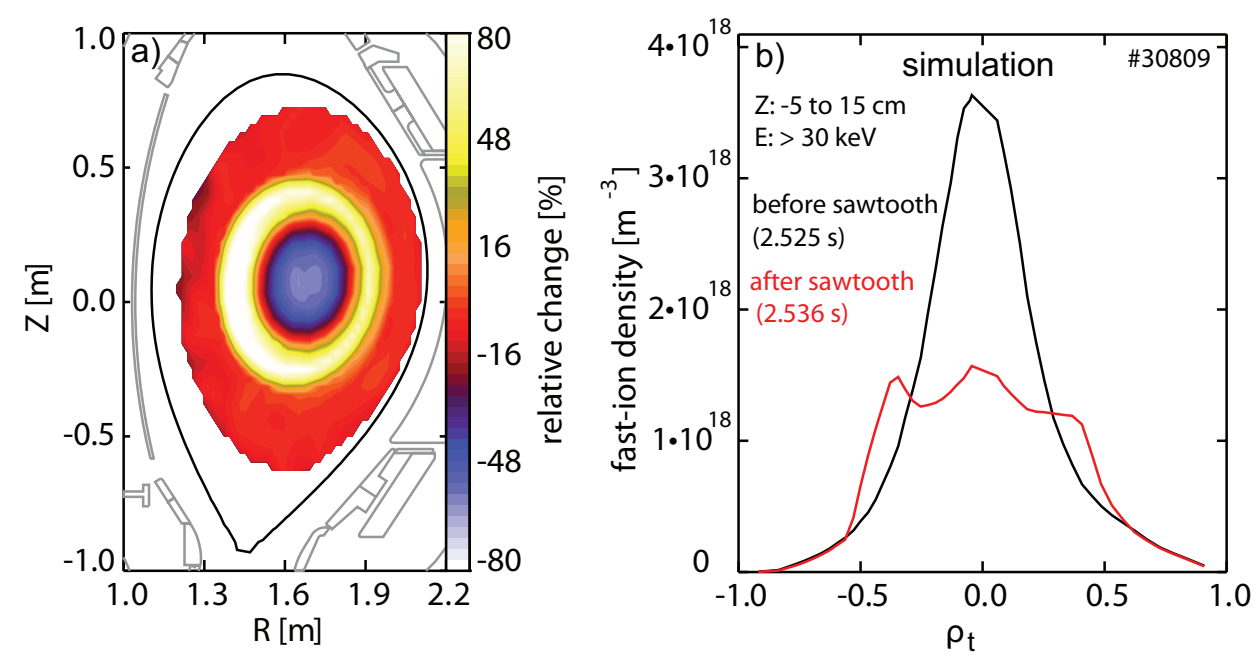

FIG. 9: a) Poloidal view on the relative change of the density of fast ions above $30 \mathrm{keV}$ due to a sawtooth crash as simulated by TRANSP using the Kadomtsev model. b) TRANSP predicted radial profiles of fast ions with energies above $30 \mathrm{keV}$ that are present between $Z=-0.05 \mathrm{~m}$ and $Z=0.15 \mathrm{~m}$ before (black) and after (red) a sawtooth crash.

timing of NBI and on the kinetic profiles. TRANSP (the module NUBEAM) first determines the deposition position of NBI and then follows the corresponding fast-ion orbits in the poloidal plane. The orbits are simulated in the gyro center approximation and the interaction with electrons and ions is considered by a Monte Carlo collision operator. The evolution of the fast-ion density is simulated by starting new fast-ion markers in the orbit simulation every $10 \mathrm{~ms}$ and by removing the thermalized fast ions as soon as their energy is below $3 / 2 \cdot T_{i}$. The sawtooth redistribution in TRANSP has been simulated by applying the Kadomtsev model [24] for predefined time points. The Kadomtsev model assumes complete reconnection of the helical magnetic field component, $\overrightarrow{B_{*}}$, which is defined relative to the helically twisted field lines in the $\mathrm{q}=1$ surface. $\overrightarrow{B_{*}}$ changes its sign at the $\mathrm{q}=1$ surface since the field lines are more twisted inside $\mathrm{q}=1$ than outside. The helical flux, i.e. the integral over $\overrightarrow{B_{*}}$ has consequently an extremum at $\mathrm{q}=1$. The model assumes that the field lines inside $\mathrm{q}=1$ reconnect with those field lines outside $\mathrm{q}=1$ that are located at the same helical flux. The new positions of the reconnected field lines after a sawtooth crash are calculated based on the conservation of the toroidal flux. Finally, the sawtooth-induced fast-ion redistribution is determined assuming that fast ions are bound to the reconnecting field lines and that their quantity is conserved.

As plotted in figures 9a and 9b, a drop of the central fast-ion density of more than $50 \%$ is simulated when applying 

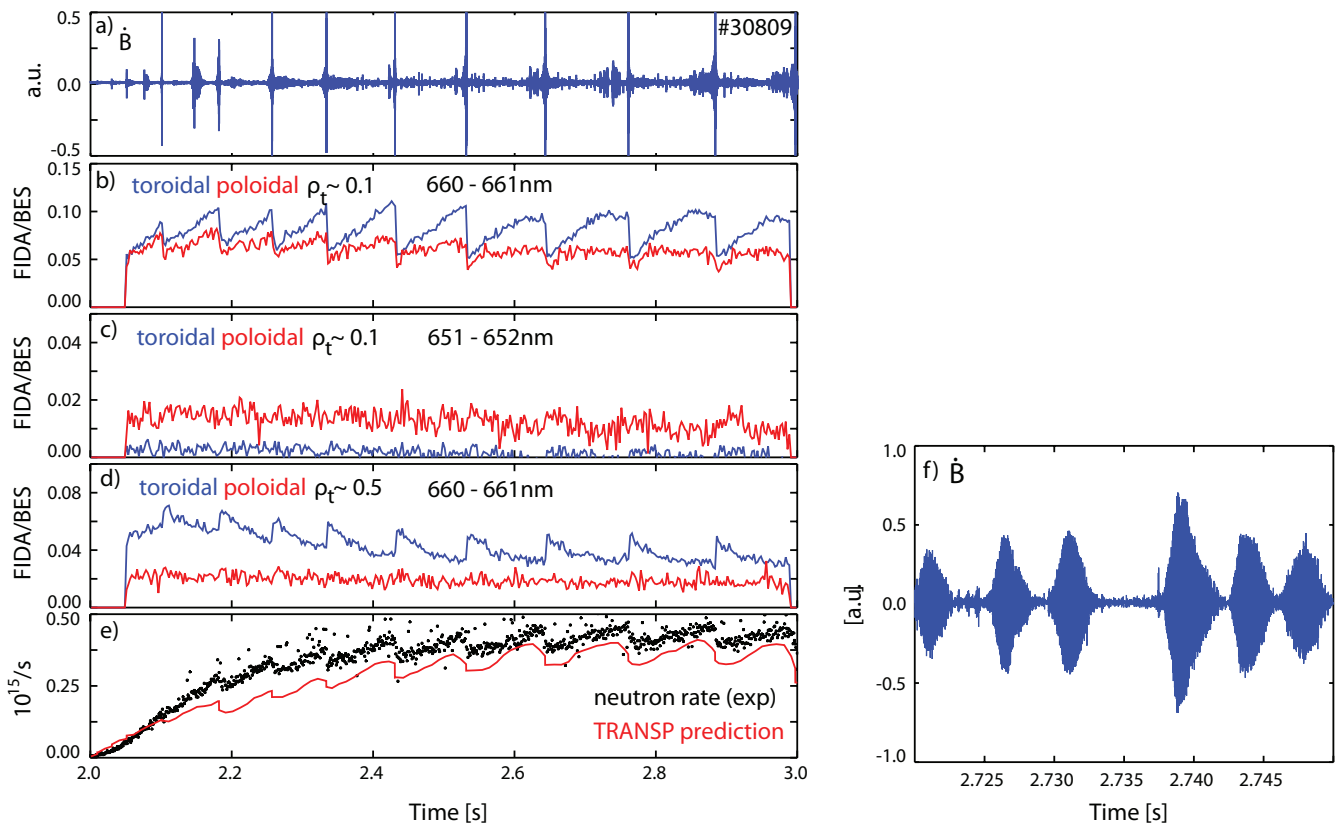

FIG. 10: a) Magnetic signal observed by a pick-up coil. b) Temporal evolution of the measured FIDA radiation from a central toroidal LOS (blue) and a central poloidal LOS (red) between $660 \mathrm{~nm}$ and $661 \mathrm{~nm}$ ( $\left.I_{\mathrm{FIDA}}\right)$, divided by the measured beam emission $\left(I_{\mathrm{BES}}\right)$. c) $I_{\mathrm{FIDA}} / I_{\mathrm{BES}}$ signal of the toroidal and poloidal LOS for wavelengths between $651 \mathrm{~nm}$ and $652 \mathrm{~nm}$. d) $I_{\mathrm{FIDA}} / I_{\mathrm{BES}}$ signal between $660 \mathrm{~nm}$ and $661 \mathrm{~nm}$ measured outside the sawtooth inversion radius. e) Measured and TRANSP predicted neutron rates. f) Zoom of figure 10a between $2.72 \mathrm{~s}$ and $2.75 \mathrm{~s}$.

the Kadomtsev model. In addition, an increase of the fast-ion density is predicted at $\rho_{\mathrm{t}} \sim 0.4$, according to the radial redistribution of fast ions. The profiles from FIDASIM in figures $8 \mathrm{a}$ and $8 \mathrm{~b}$ represent this TRANSP simulation. Their good agreement with the measurements gives evidence that the sawtooth crashes internally redistribute a large part of the confined fast ions.

In figure 10e, the temporal evolution of the measured neutron rates (black) is compared with the neutron flux predicted by the TRANSP code (red). The drops of the predicted neutron rates after every sawtooth crash are in good agreement with the measurement. The relatively weak variation by only about $10 \%$ (compared to a fast-ion redistribution of about 50\%) agrees with the observation from FIDA spectroscopy: the sawtooth crashes mainly cause an internal fast-ion redistribution but no significant losses. Indeed, if the sawteeth ejected fast ions from the plasma, the neutron rates would drop more significantly. Here, it should be noted that the absolute value of the neutron rate is not perfectly matched by the simulation which may be explained by uncertainties in the measured effective charge. The latter leads to uncertainty in the deuterium density which yields uncertainty in the calculation of neutron rates: the fusion reaction between the fast ions and thermal deuterium ions is typically the dominant source of neutrons.

In addition to the neutron rates, figure 10 compares the temporal evolution of the FIDA data from the toroidal and poloidal LOS with the measurement of a magnetic pick-up coil. The FIDA data represents the integrated FIDA radiation (in wavelength), divided by the level of the beam emission which is measured in the spectra of the toroidal LOS (see figure 4). The beam emission depends on the attenuation of the injected neutrals and, hence, allows us to account for changes of the density of neutrals present along the NBI path. The latter could modify the FIDA signal even if the fast-ion density was constant. The ratio between the FIDA radiation, $I_{\mathrm{FIDA}}$, and the beam emission, $I_{\mathrm{BES}}$, does account for this effect and is a more appropriate signal to study the temporal evolution of the fast-ion density. The $I_{\text {FIDA }} / I_{\text {BES }}$ signals from a central toroidal (blue) and poloidal (red) LOS are shown in figure 10b for red-shifted wavelengths (between $660 \mathrm{~nm}$ and $661 \mathrm{~nm}$ ). Clearly, the measured signals drop simultaneously with the appearance of the sawtooth crashes in the magnetic signal and then recover slowly. Interestingly the $I_{\mathrm{FIDA}} / I_{\mathrm{BES}}$ signals from the two viewing geometries first show a similar trend which then becomes different from about $2.5 \mathrm{~s}$ on: While the signal from the toroidal LOS increases monotonically between the crashes, the $I_{\text {FIDA }} / I_{\text {BES }}$ signal from the poloidal view saturates. This different behavior might be explained by the rise of the electron and impurity density during the observed time interval and, hence, by the increase of the collision frequency. Increased collision frequencies cause 
stronger pitch angle scattering and consequently a more efficient redistribution of energetic particles to passing orbits to which the toroidal views are very sensitive (see figure 5). In contrast, the poloidal views measure a saturated density as these views are sensitive to fast ions at pitch values in the broad vicinity of 0.5 which corresponds to the injection geometry of NBI Q3. For these ions to exist, no pitch angle scattering is necessary. Pitch angle scattering in fact rather reduces the fast-ion density in the observed region of velocity space. The data shown in figure 10c corresponds the blue shifted wavelengths, i.e. mainly counter-rotating fast ions. The $I_{\mathrm{FIDA}} / I_{\mathrm{BES}}$ signal from the poloidal LOS (red) covers the velocity space of trapped fast ions. Interestingly, the measurement shows no correlation with the appearance of the sawtooth crashes, indicating that the sawtooth-induced fast-ion redistribution is weak in the observed part of velocity space. It should be noted that the toroidal measurement (blue) exhibits almost no FIDA signal. The density of purely counter-rotating fast ions, probed by this view at blue shifted wavelengths, is negligible because the NBI geometry is co-current.

The $I_{\text {FIDA }} / I_{\mathrm{BES}}$ signals measured outside the $\mathrm{q}=1$ surface (at $\rho_{\mathrm{t}}=0.5$ ) are plotted in figure 10d for red-shifted wavelengths. Clearly, the toroidal LOS measures an increase of the $I_{\text {FIDA }} / I_{\mathrm{BES}}$ signal directly after the sawtooth crashes, followed by a slow decay within about $50 \mathrm{~ms}$. This shows that passing fast ions are radially redistributed by the crashes and that these ions are subsequently slowed down. In contrast to this observation, the poloidal data outside $q=1$ does not exhibit an increase after the crashes. This is in agreement with the observation of a weaker redistribution of the fast ions in the broad vicinity of pitch values of 0.5 . Moreover, the absence of an increased signal can be explained since the redistributed fast ions change their pitch angle. Fast particles that are ejected to the low-field side keep their magnetic moment and, hence, get on more passing orbits. These more passing orbits are rarely detected by the poloidal views while the toroidal LOS are very sensitive to such modification of the pitch angle distribution.

The clear observation of the fast-ion redistribution due to sawtooth crashes demonstrates the high sensitivity and good signal to noise ratio of the FIDA diagnostic. Therefore it is possible to also study the fast-ion transport induced by other instabilities such as fishbones. Fishbone instabilities are well visible in discharge \#30809 before the sawtooth crashes from about $2.5 \mathrm{~s}$ on. Figure $10 \mathrm{f}$ shows a zoom of the magnetic data plotted in figure $10 \mathrm{a}$ between $2.72 \mathrm{~s}$ and $2.75 \mathrm{~s}$. The increasing and then decreasing oscillations in the magnetic measurement are a clear footprint of fishbone activity. The modes are firstly excited by the resonant interaction with trapped fast ions. The subsequent decay of the mode amplitude is then explained by anomalous fast-ion transport: The instability redistributes the resonant particles in phase space, loses its drive and, consequently, decays. This anomalous fast-ion transport is, however, relatively small or localized in a very specific part of the phase space. Neither the measured neutron rates, nor the FIDA data exhibit a variation that is correlated with the presence of the individual fishbone modes. Therefore, we can state that, under the investigated conditions, the fishbone instability is a small perturbation of the fast-ion distribution function. In particular, a strong anomalous fast-ion transport of the passing fast ions can be excluded because these particles are very well probed. In order to obtain a better signal to noise ratio in the measurement of the trapped fast-ion population, new experiments are planned using more radial NBI geometry. This produces more trapped fast particles and increases the drive of the fishbone modes.

The experimental evidence of a very strong sawtooth-induced redistribution of co-rotating fast ions, as presented here, is in good agreement with recent observations at ASDEX Upgrade [19]. There also, a sawtooth-induced increase of the fast-ion density outside the sawtooth inversion radius has been observed that is followed by a relatively slow decay. Good agreement between the corresponding decay times and the fast-ion slowing down times hereby suggests that the fast-ion transport is neo-classical in the phase between the crashes.

\section{MHD-QUIESCENT EXPERIMENTS USING ON- AND OFF-AXIS NBI}

\begin{tabular}{|l|l|l|l|l|l|}
\hline $\mathrm{B}_{\mathrm{t}}$ & $\mathrm{I}_{\mathrm{p}}$ & $\mathrm{n}_{\mathrm{e}}$ & $\mathrm{q} 95$ & $\rho_{\mathrm{t}}(\mathrm{q}=1)$ & $\delta \mathrm{B}_{\max }$ \\
\hline$-2.5 \mathrm{~T}$ & $0.8 \mathrm{MA}$ & $6.9 \times 10^{19} \mathrm{~m}^{-3}$ & 5.27 & 0.15 & $3 \mathrm{mT}$ \\
\hline
\end{tabular}

TABLE II: Main discharge parameters of \#27237.

As already mentioned in the introduction, previous experiments at ASDEX Upgrade suggested that small scale turbulence is responsible for the observation of weaker off-axis NBI current drive than predicted by neo-classical theory. Motivated by our new diagnostic capabilities, MHD-quiescent discharges, similar to these prior experiments 

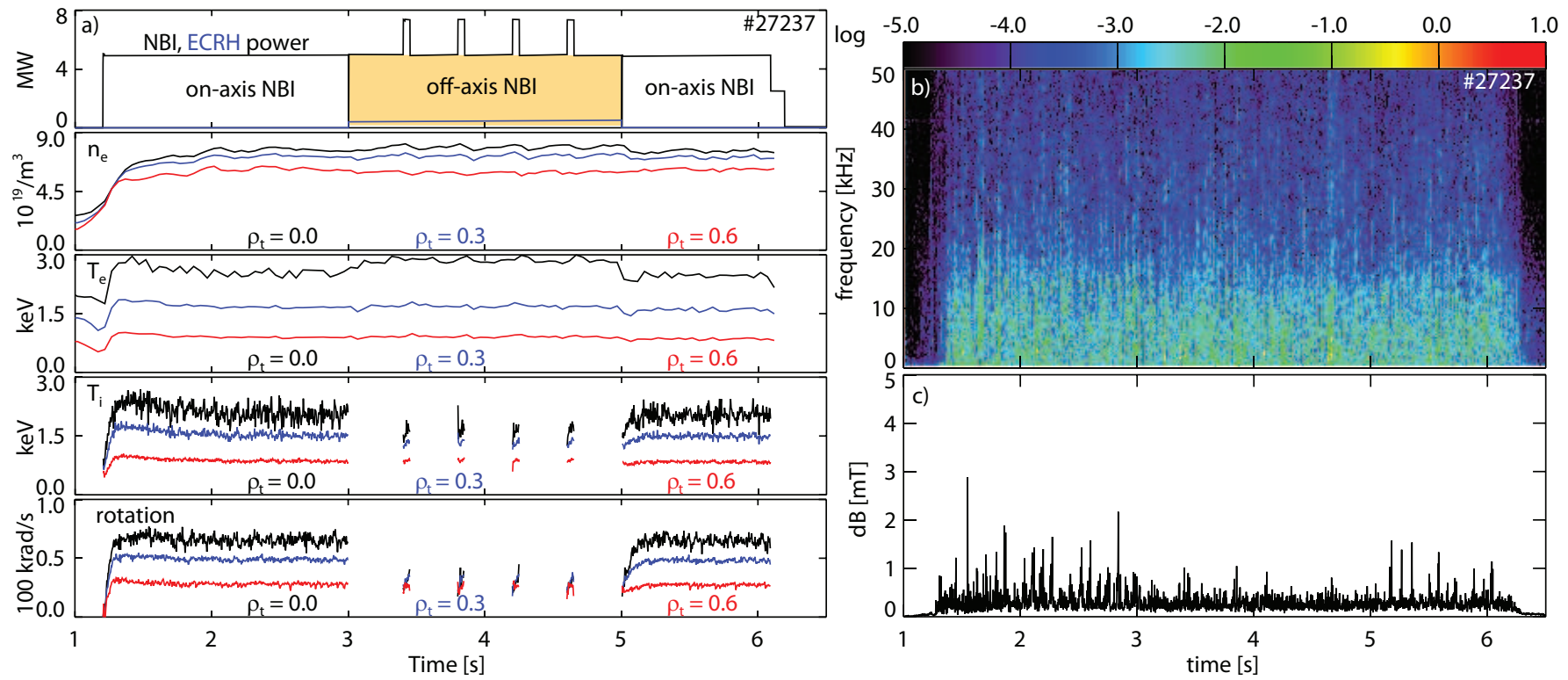

FIG. 11: a) Characteristic time traces of discharge \#27237 showing the NBI and ECRH heating power, the electron density and temperature, the ion temperature and the toroidal plasma rotation. b) Magnetic spectrogram of discharge \#27237. c) Approximate magnetic perturbation amplitude of $n=1, m=1$ modes activity based on measurements of a magnetic pick-up coil.

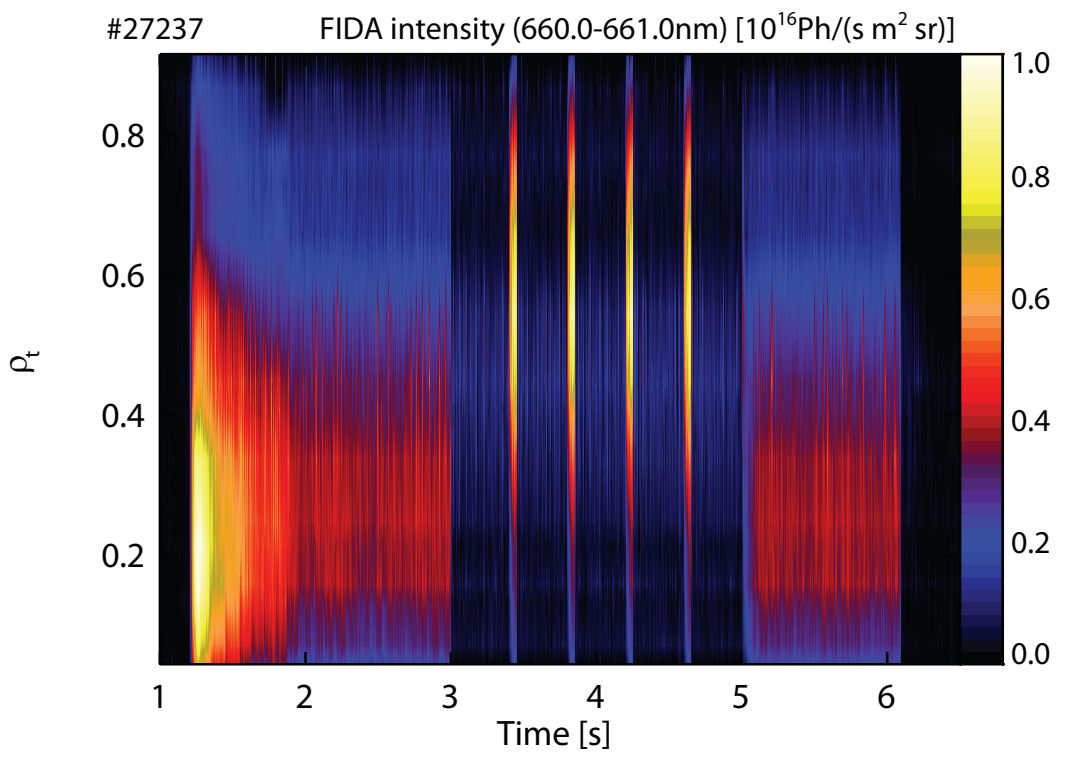

FIG. 12: Temporal evolution of radial FIDA intensity profiles as a function of the normalized toroidal flux. The profiles correspond to the FIDA radiation, observed by the toroidal LOS that has been integrated between $660 \mathrm{~nm}$ and $661 \mathrm{~nm}$.

were performed with a toroidal magnetic field of $-2.5 \mathrm{~T}$ and a plasma current of $800 \mathrm{kA}$ (see table II). Characteristic time traces of a representative discharge (\#27237) are plotted in figure 11a. $5 \mathrm{MW}$ of on-axis NBI heating power was replaced by off-axis NBI between $3 \mathrm{~s}$ and $5 \mathrm{~s}$, simultaneously with the application of electron cyclotron resonance heating $(\mathrm{ECRH})$ to maintain a constant central electron temperature. In addition, short pulses from NBI Q3 were made as the FIDA and CXRS measurements rely on that beam line. Thus, ion temperature and rotation measurements can be performed between $3 \mathrm{~s}$ and $5 \mathrm{~s}$. A significant drop of the central ion temperature and rotation is observed during this off-axis NBI phase, indicating that the heat and momentum deposition profile of the fast ions has changed. This is in agreement with the temporal evolution of radial FIDA intensity profiles from the toroidal LOS, as plotted in figure 12. The shape of the profiles clearly changes when switching from on-axis NBI to off-axis NBI heating. Between 

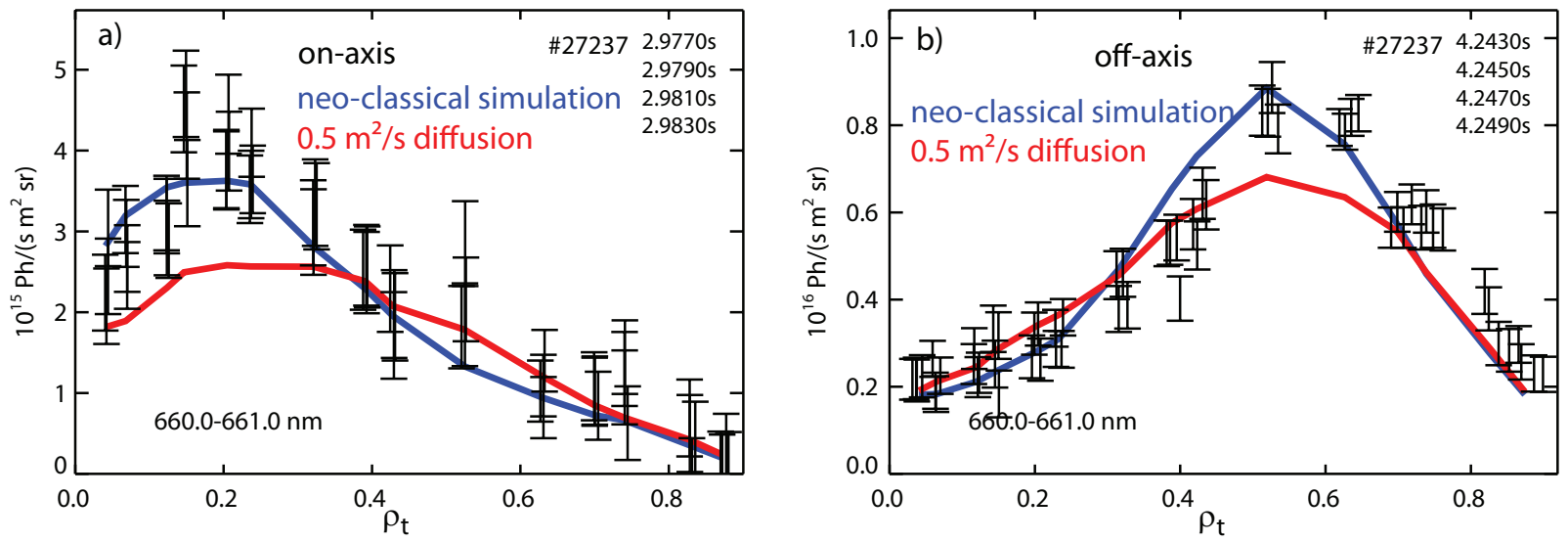

FIG. 13: Radial FIDA intensity profiles (between $660 \mathrm{~nm}$ and $661 \mathrm{~nm}$ ) measured during on-axis NBI (a) and during off-axis NBI (b) in discharge \#27237 for four successive time intervals. The passive radiation, measured during the phase without operation of NBI Q3, has been subtracted. The error-bars correspond to the statistical noise in the spectra. In blue, simulated profiles from FIDASIM are shown that correspond to a neo-classical fast-ion distribution function from TRANSP. In red, a simulation based on an anomalous fast-ion transport of $0.5 \mathrm{~m}^{2} / \mathrm{s}$ is plotted.

$1.2 \mathrm{~s}$ and $3 \mathrm{~s}$ and between $5 \mathrm{~s}$ and $6 \mathrm{~s}$, the profiles are peaked at $\rho_{\mathrm{t}} \approx 0.3$ which corresponds to the on-axis NBI injection scheme. During the application of off-axis NBI (between $3 \mathrm{~s}$ and $5 \mathrm{~s}$ ) the active FIDA radiation, only visible when NBI Q3 is operational, is peaked at $\rho_{\mathrm{t}} \approx 0.5$. Here, it should be noted that the intensity of the FIDA radiation not only depends on the fast-ion density, but also on the density of neutrals along NBI Q3. The latter is attenuated towards the plasma center and, thus, the FIDA profiles are not peaked in the very center during on-axis NBI heating but slightly off-axis. Forward modeling by FIDASIM is consequently needed to interpret the absolute value and shape of the FIDA profiles. Figure 13a shows radial FIDA intensity profiles that were measured during on-axis NBI at about $2.98 \mathrm{~s}$ and figure $13 \mathrm{~b}$ shows profiles measured during off-axis NBI at about $4.25 \mathrm{~s}$. Passive radiation has been subtracted from the data, based on measurements when NBI Q3 was off. In addition to the experimental data, FIDASIM results are shown for two different fast-ion slowing down distributions from TRANSP. The simulated radial FIDA intensity profiles in blue represent a neo-classical fast-ion behavior while the profiles in red are calculated with the assumption of a constant (in time and space) anomalous fast-ion diffusion of $0.5 \mathrm{~m}^{2} / \mathrm{s}$. The radial FIDA intensity profiles measured during on-axis NBI, but also those measured during off-axis NBI are in good agreement with the neo-classical simulation. The assumption of an additional fast-ion transport does, on the contrary, not fit most parts of the experimental data. This indicates that, in the presence of weak MHD activity (see figures 11b and 11c), the anomalous fast-ion diffusion is well below $0.5 \mathrm{~m}^{2} / \mathrm{s}$. The effect of small scale turbulence on the radial fast-ion transport is consequently small.

It should be noted that the detailed investigation of the current driven by the application of on- and off-axis NBI in discharge \#27237 is not possible. The most important diagnostic to study of the current distribution at AUG, i.e. the MSE diagnostic, relies on the continuous operation of NBI Q3. Since this beam source was only operated in short blips during off-axis NBI, the evolution of the current profile cannot be analyzed. For this reason, new NBI current drive experiments have been conducted in the 2014 experimental campaign. The results of these experiments are presented in the next section.

\section{OFF-AXIS NBI CURRENT DRIVE EXPERIMENTS USING 10 MW OF HEATING POWER}

\begin{tabular}{|l|l|l|l|l|l|}
\hline $\mathrm{B}_{\mathrm{t}}$ & $\mathrm{I}_{\mathrm{p}}$ & $\mathrm{n}_{\mathrm{e}}$ & $\mathrm{q} 95$ & $\rho_{\mathrm{t}}(\mathrm{q}=1)$ & $\delta \mathrm{B}_{\max }$ \\
\hline$-2.6 \mathrm{~T}$ & $0.8 \mathrm{MA}$ & $5.3 \times 10^{19} \mathrm{~m}^{-3}$ & 5.38 & 0.15 & $10 \mathrm{mT}$ \\
\hline
\end{tabular}

TABLE III: Main discharge parameters of \#30840.

The analysis of the NBI current-drive (NBCD) efficiency is closely linked to the study of the transport of beam- 



FIG. 14: a) Characteristic time traces of discharge \#30840 showing the NBI and ECRH heating power, the electron density, the effective charge, the electron and ion temperatures and the toroidal plasma rotation. The kinetic data is plotted for three different radial positions. b) Spectrogram of a magnetic pick-up coil showing the footprints of fishbone instabilities (i.e. the frequency chirping between $15 \mathrm{kHz}$ and $25 \mathrm{kHz}$ ) and that of a continuous mode at the $\mathrm{q}=1$ surface between $4 \mathrm{~s}$ and $7 \mathrm{~s}$. c) Approximate magnetic perturbation induced by these modes based on measurements of a magnetic pick-up coil.

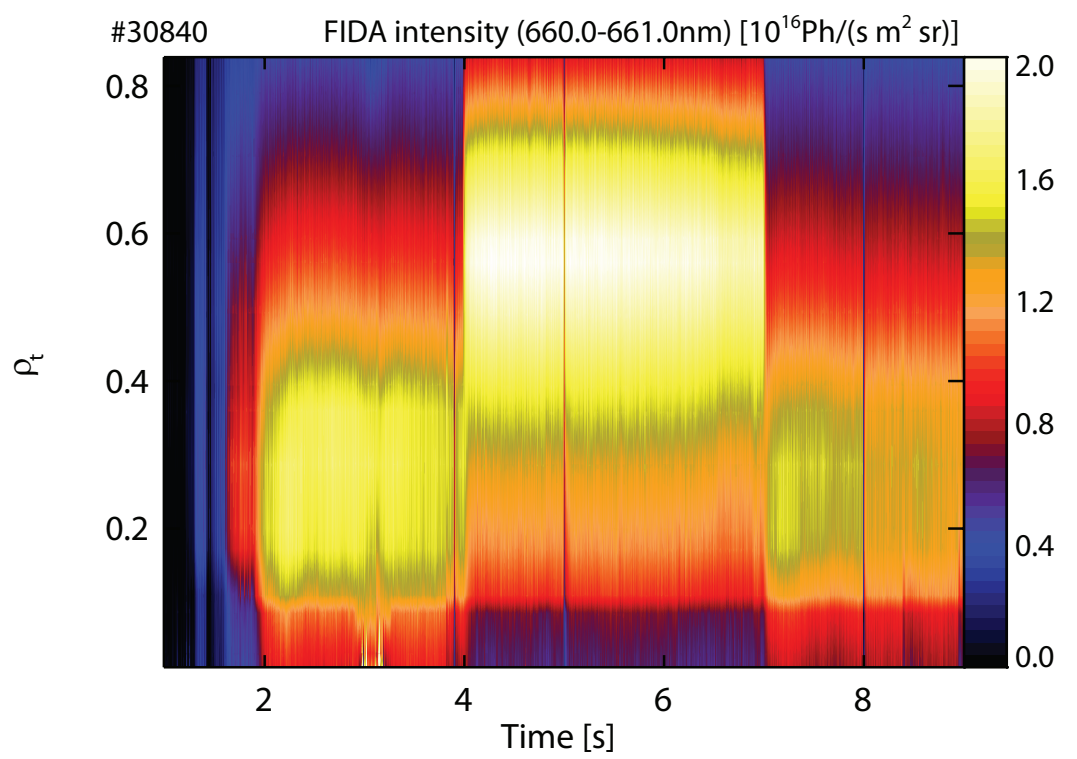

FIG. 15: Temporal evolution of radial FIDA intensity profiles as a function of the normalized toroidal flux. The profiles correspond to the FIDA radiation, observed by the toroidal LOS that has been integrated between $660 \mathrm{~nm}$ and $661 \mathrm{~nm}$.

generated fast ions: If fast ions were redistributed by an anomalous diffusion, also the current driven by these ions would change. Consequently, the combined analysis of the fast-ion density and the fast-ion driven current can provide a more detailed view on the fast-ion transport processes. Moreover, the analysis of NBCD is a very important topic for future fusion devices since the continuous operation of tokamak discharges, as well as the capability to tailor the q-profile, relies in part on efficient NBCD. In the 2014 experimental campaign, discharges with $-2.6 \mathrm{~T}$ of toroidal magnetic field and a plasma current of $800 \mathrm{kA}$ have been performed to study the effect of the off-axis NBI sources on the fast-ion and current profiles (see table III). Characteristic time traces of a representative discharge (\#30840) 

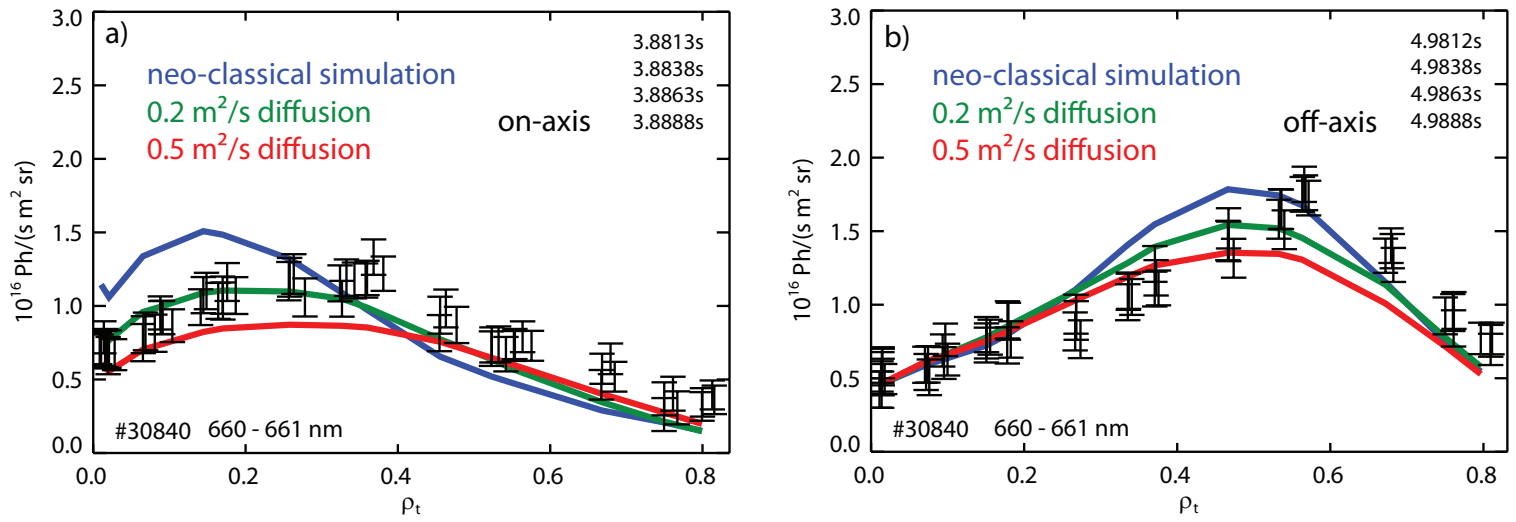

FIG. 16: Measured and simulated radial FIDA profiles during on-axis NBI (a) and during off-axis NBI (b). In blue, a neoclassical simulation is shown. A simulation based on an anomalous fast-ion transport of $0.2 \mathrm{~m}^{2} / \mathrm{s}$ is plotted in green. In red, a simulation is given that is based on an anomalous fast-ion transport of $0.5 \mathrm{~m}^{2} / \mathrm{s}$.
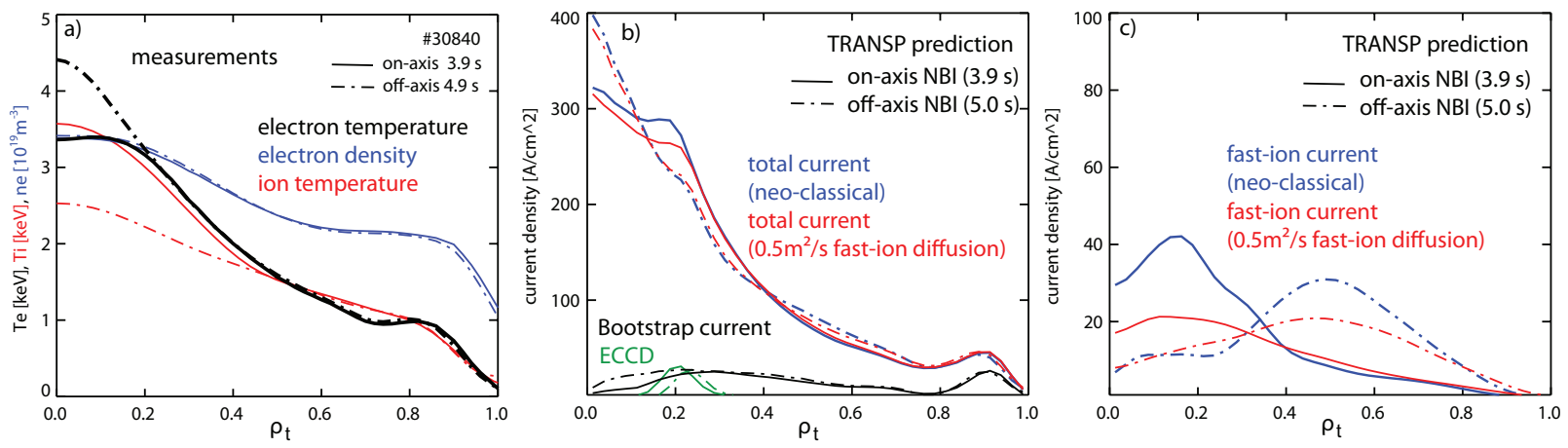

FIG. 17: a) Measured electron temperature (black), ion temperature (red) and electron density (blue) profiles during on-axis NBI (solid lines) and during off-axis NBI (dashed lines) b) Calculated current density profile during on-axis NBI (solid lines) and during off-axis NBI (dashed lines) of the total current (blue), the Bootstrap current (black) and the current driven by the application of central ECCD (green). In addition the total current is shown when assuming anomalous fast-ion transport. c) Predicted fast-ion current density from NBI for the on-axis and off-axis cases. In blue, the neo-classical simulation is plotted. In red, the simulation assuming an anomalous fast-ion diffusion of $0.5 \mathrm{~m}^{2} / \mathrm{s}$ is shown.

are shown in figure 14a. As can be seen, $7.5 \mathrm{MW}$ of NBI heating power were applied from $2 \mathrm{~s}$ until $9 \mathrm{~s}$. To provide continuous FIDA, CXRS and MSE measurements, NBI Q3 was turned on during that whole phase. In addition, three $3 \mathrm{~s}$ long phase of $5 \mathrm{MW}$ of on-axis NBI (Q5 and Q8) and of off-axis NBI (Q6 and Q7) where applied. These relatively long phases are needed to study the evolution of the current profile: When turning on a given current drive source, the injected net current creates a magnetic field that generates a second mirror current carried by electrons. Both currents are firstly compensating each other until the mirror current decays on the relatively long resistive time scale of few seconds.

Additionally to the application of NBI heating, electron cyclotron current drive (co-ECCD) was used in the plasma center throughout the discharge in order to avoid sawtooth crashes. The latter could interfere with our measurements because sawteeth broaden the fast-ion and current profiles. Moreover, $0.6 \mathrm{MW}$ of modulated $(30 \mathrm{~Hz})$ preemptive ECCD was successfully applied at the $q=1.5$ surface to prevent the possible destabilization of a tearing mode that could also affect our measurements. When replacing two on-axis NBI sources by the off-axis sources between $4 \mathrm{~s}$ and $7 \mathrm{~s}$, additional central ECRH is applied to keep the temperature profile constant. The ohmic current profile strongly depends on the plasma resistivity and, hence, on the electron temperature profile. If the central electron temperature dropped, the current profile would broaden, even without the influence of fast particles. To maintain constant electron temperature profiles, a new real-time algorithm was developed and applied for the first time in this experiment. The algorithm receives the electron temperature measured by the ECE diagnostic at $\rho_{\mathrm{t}}=0.2$ and accordingly controls the ECRH power. As can be seen in figure 17a, the feedback-controlled application of central 
ECRH results in comparable electron temperature profiles during on- and off-axis NBI from $\rho_{\mathrm{t}}=0.2$ outwards. In the very center, slightly too high electron temperatures are measured which is, however, not crucial of the analysis of the NBI current drive efficiency: The plasma volume is small for $\rho_{\mathrm{t}}<0.2$ and too high temperatures yield more peaked current profiles which is opposite to what we expect from off-axis NBI current drive. The electron density, as well as the effective charge (determined from the level of Bremsstrahlung in the spectra of the CXRS diagnostics [32]) remains unchanged between $2 \mathrm{~s}$ and $9 \mathrm{~s}$. In contrast, the central ion temperature and rotation (which both do not impact the current profile) clearly drop when switching to off-axis NBI, explained by the different torque and heat deposition profiles of off-axis NBI.

The temporal evolution of the radial FIDA intensity profiles is plotted in figure 15. The modification of the FIDA profiles is clearly observed when switching from on-axis NBI to off-axis NBI: During on-axis NBI, the radial intensity profiles are peaked at $\rho_{\mathrm{t}} \approx 0.2$ (the FIDA intensity profiles do not peak in the plasma center as they also depend on the attenuation of NBI Q3) and during off-axis NBI, the maximum of the radial FIDA intensity profiles is located at $\rho_{\mathrm{t}} \approx 0.5$.

In figures $16 \mathrm{a}$ and $16 \mathrm{~b}$ the experimental profiles are plotted in black for four successive time points during on-axis NBI (at 3.88 s) and during off-axis NBI (at $4.98 \mathrm{~s}$ ). The profiles correspond to spectra from the toroidal LOS that were integrated between $660 \mathrm{~nm}$ and $661 \mathrm{~nm}$ and from which passive radiation was subtracted. In addition, simulated profiles (FIDASIM+TRANSP) are shown in color. The profiles plotted in blue represent the FIDA radiation expected from neo-classical fast-ion distribution functions from TRANSP (already presented in section II, figures 2 and 3 ). In addition, figures $16 \mathrm{a}$ and $16 \mathrm{~b}$ show profiles plotted in red and green that represent simulations assuming constant anomalous transport of $0.2 \mathrm{~m}^{2} / \mathrm{s}$ and $0.5 \mathrm{~m}^{2} / \mathrm{s}$, respectively. This very basic assumption of anomalous fast-ion diffusion is a 0 -order approach to assess the effect of instabilities on the fast-particle confinement. However, a relatively good agreement between the measured profiles and the simulation assuming an anomalous fast-ion diffusion of $0.2 \mathrm{~m}{ }^{2} / \mathrm{s}$ is found. This indicates a finite, but small anomalous transport and we can define an approximate upper boundary of the anomalous fast-ion transport in discharge \#30840: $0.5 \mathrm{~m}^{2} / \mathrm{s}$.

It should be noted that, despite the anomalous fast-ion diffusion of up to $0.5 \mathrm{~m}^{2} / \mathrm{s}$, the radial fast-ion density profiles change when switching from on-axis NBI to off-axis NBI. This consequently changes the current driven by the on- and off-axis fast-ion populations. Figures $17 \mathrm{~b}$ and $17 \mathrm{c}$ illustrate the TRANSP predicted contributions to the current profile (except the ohmic current). The solid lines correspond to the on-axis NBI phase while the dashed lines represent the off-axis NBI heating case. The contribution from the bootstrap current is plotted in black in figure 17b. It is nearly unaffected when comparing the on-axis phase with the off-axis phase because the electron density and temperature profiles remain roughly constant during the discharge. Also the current generated by the application of ECCD, visible in green, does not vary appreciably. The total current profile (blue) changes when replacing on-axis NBI by off-axis NBI because the fast-ion current density changes. The NBI driven current, as calculated by TRANSP using [33], is shown in figure $17 \mathrm{c}$ in blue. It varies significantly when switching from on-axis NBI to off-axis NBI. In addition to this neo-classical prediction, simulated current density profiles are given in red that represent an anomalous fast-ion diffusion of $0.5 \mathrm{~m}^{2} / \mathrm{s}$ (the upper boundary of the fast-ion transport observed by FIDA spectroscopy). As can be seen, the anomalous fast-ion diffusion results in a reduction of the fast-ion driven current by up to $50 \%$.

In the following, this prediction is compared with the experimental data from DCN polarimetry, magnetic signals (the internal inductance) and with data from the MSE diagnostic. The MSE diagnostic analyzes a part of the beam emission spectrum emitted by fully energetic neutrals from NBI Q3 (the sigma component of D-alpha light that is split by the Stark effect). The polarization direction of this radiation depends on the projection angle of the magnetic field lines on the plane perpendicular to the MSE's LOS and is sensitive to changes of the poloidal magnetic field. It, hence, allows us to investigate the plasma current distribution. In figure 18, measured projection angles from five LOS of the MSE diagnostic are plotted in gray. The individual LOS measure at different radial positions which are indicated by values of the normalized toroidal flux. A clear variation of the MSE measurement is visible at $3 \mathrm{~s}$. This change is caused by a radial sweep of the plasma column by about $2.5 \mathrm{~cm}$ that was performed to validate the measurement. By sweeping the plasma, different field line angles are scanned over the LOS. The clear effect of the sweep proves that the diagnostic measures changes of the field line angles accurately. When replacing the on-axis NBI sources by the off-axis sources in discharge \#30840 at $4 \mathrm{~s}$, a fast change of the field line angles is observed in the outer LOS. This change is related to a change of the pressure profile: When switching from on-axis NBI heating to off-axis NBI, the ion and fast-ion pressure profiles change on the time scale of the fast-ion slowing-down time which is in the range of $40 \mathrm{~ms}$ in discharge \#30840. This change results in a different Shafranov shift of the plasma center (about $1.5 \mathrm{~cm}$ as predicted by TRANSP) and, thus, in a relatively fast change of the angles observed by the MSE. After this 


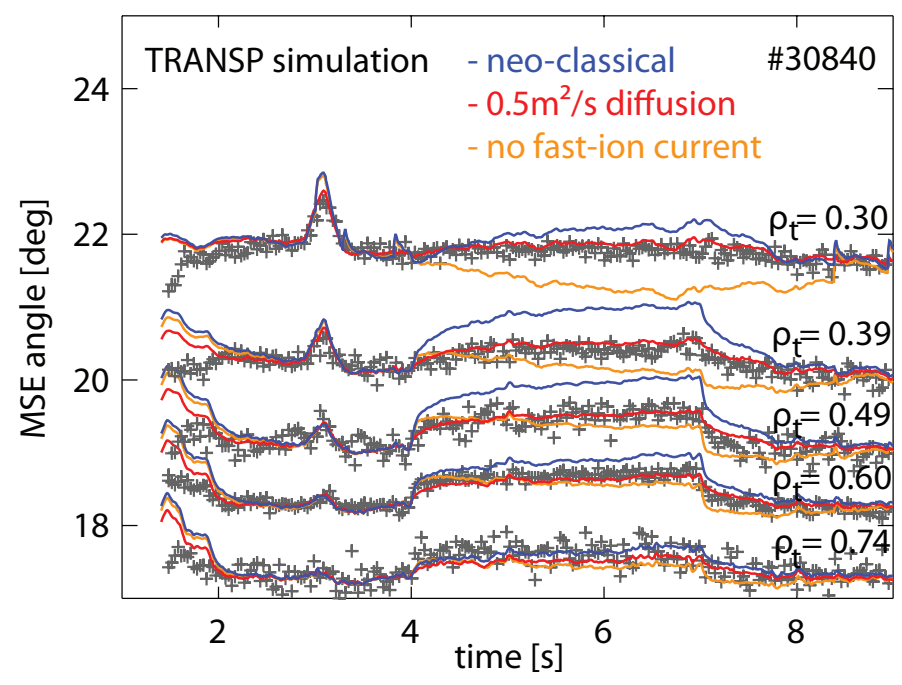

FIG. 18: Time traces of measured MSE angles during discharge \#30840. The experimental data is compared with simulations from TRANSP assuming a neo-classical fast-ion behavior (blue), an anomalous fast-ion diffusivity of $0.5 \mathrm{~m}^{2} / \mathrm{s}$ (red) and no current drive from the fast ions (orange).
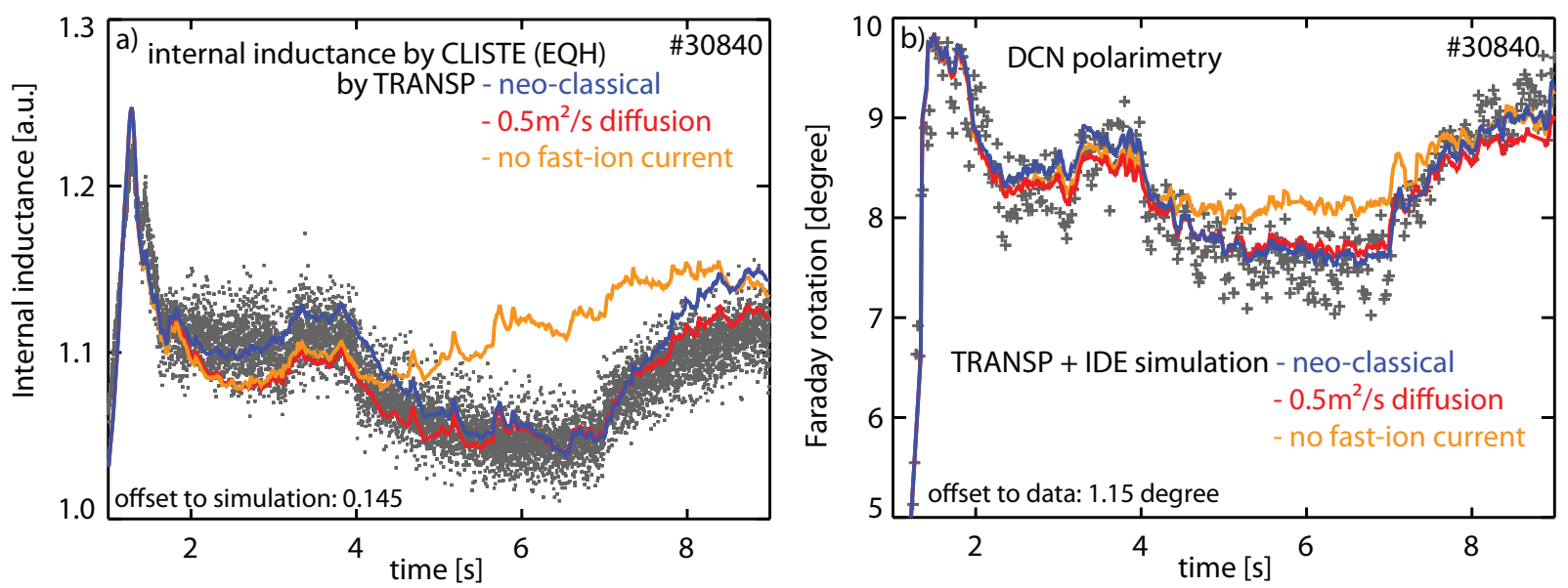

FIG. 19: Time traces of the internal inductance as reconstructed by CLISTE (a) and measured polarization degree of a DCN laser that propagates horizontally through the plasma (b). The experimental data is compared with simulations from TRANSP assuming a neo-classical fast-ion behavior (blue), an anomalous fast-ion diffusivity of $0.5 \mathrm{~m}^{2} / \mathrm{s}$ (red) and no current drive from the fast ions (orange).

step, a slow and continuous evolution is visible in the outer channels of MSE diagnostic that can be attributed to the off-axis NBI current drive.

In color, simulated MSE angles from TRANSP are plotted that are normalized to the measured data before the off-axis NBI heating phase. The variation of the measured MSE angles during the radial sweep is well matched by the simulation. In blue, the neo-classical simulation is shown that predicts much stronger changes of the MSE angles than actually observed. The data in red represents a TRANSP simulation assuming an anomalous fast-ion diffusion of $0.5 \mathrm{~m}^{2} / \mathrm{s}$. This simulation well agrees with the data. The assumption that the fast ions do not drive current at all (orange) does not fit.

This result is in agreement with investigations of the internal inductance $\left(\ell_{\mathrm{i}}\right)$. The internal inductance depends on the volume integral of the square of the poloidal magnetic field inside the separatrix [34] and is high when the current profile is peaked in the plasma center and small when it is broad. It can be reconstructed by equilibrium codes that are based on magnetic coil measurements which yield $\ell_{\mathrm{i}} / 2+\beta_{\mathrm{pol}}$ [35] where $\beta_{\mathrm{pol}}$ is the ratio of the plasma pressure and the poloidal magnetic field energy. The internal inductance based on magnetic measurements has been 
reconstructed by the CLISTE equilibrium code [36] and is shown in gray in figure 19. It clearly shows the effect of the off-axis NBI sources between $4 \mathrm{~s}$ and $7 \mathrm{~s}$ that shift the current distribution outwards, causing the reduction of $\ell_{\mathrm{i}}$. The slow decrease during off-axis NBI heating and the subsequent increase after switching back to on-axis NBI at $7 \mathrm{~s}$ can be explained by the long current diffusion times $(\sim 1 \mathrm{~s})$. In blue, the time trace of the simulated $\ell_{\mathrm{i}}$ (TRANSP) is illustrated that is calculated from the predicted current distribution assuming a neo-classical fast-ion behavior (a constant offset of 0.145 has been added to the simulations in order to match the measurement, explained by slightly different definitions of $\ell_{\mathrm{i}}$ in the codes). The temporal evolution of the simulation matches the measurement well. Also the simulated $\ell_{\mathrm{i}}$ that corresponds to an anomalous fast-ion transport of $0.5 \mathrm{~m}^{2} / \mathrm{s}$, shown in red, is consistent with the $\ell_{\mathrm{i}}$ from CLISTE. Assuming no fast-ion driven current, however, clearly does not match. Also measurements yielded by a novel polarimetry diagnostic [22] show a clear effect of off-axis neutral beam current drive. The polarimetry diagnostic measures the Faraday rotation of the polarization direction of a DCN laser beam that propagates horizontally through the plasma at about mid radius. The Faraday rotation is sensitive to the poloidal magnetic field and, hence, allows us to investigate the plasma current distribution. Figure 19b shows in gray the measurement of the polarimetry diagnostic compared with a forward model (called IDE) that uses TRANSP predicted equilibria. The simulated polarimetry data, which corresponds to a neo-classical TRANSP simulation/equilibrium, is plotted in blue. In red, a simulation is plotted, which assumes an anomalous fast-ion transport of $0.5 \mathrm{~m}^{2} / \mathrm{s}$. The simulation illustrated in orange corresponds to a prediction assuming no fast-ion driven current. It should be noted that the experimental data has been corrected by a constant offset (1.45 degrees, likely explained by uncertainties in the offset determination of the DCN diagnostic). Moreover, the present polarimeter setup shows increased noise level during density ramps. In the time window of interest of discharge \#30840, however, the density was constant and the uncertainty in the polarimetry signal therefore small. We can consequently conclude that the simulation plotted in orange, i.e. the assumption that no current is driven by the fast ions, does not match the experiment.

In conclusion, the overall agreement of four independent measurements shows that the application of off-axis NBI produces a clear change of the radial fast-ion profiles. Not only is the FIDA radiation shifted outwards during offaxis NBI. Also the current profile is modified when switching from on-axis NBI to off-axis NBI. Predictions from TRANSP that assume an anomalous fast-ion diffusion of up to $0.5 \mathrm{~m}^{2} / \mathrm{s}$ are in agreement with all measurements and, hence, show that under the investigated conditions, a weak fast-ion transport is present. It can be speculated if this anomalous transport is caused by turbulence. However, also MHD instabilities might be responsible for the mismatch between the experimental data and the neo-classical simulations. Despite the suppression of large tearing modes in discharge \#30840, the magnetic spectrogram, plotted in figure 14b, indicates fishbone activity during the on-axis phases and a continuous $n=1, m=1$ mode during the off-axis phase. An effect of these modes on the fast-ion confinement cannot be excluded which makes a clear conclusion on the origin of the anomalous transport impossible. However, it should be noted that these results imply that in future fusion devices, the application of off-axis NBI current drive is likely to be effective to tailor and modify the current profiles.

\section{CONCLUSION}

The impact of sawtooth crashes on the distribution function of fast ions with $60 \mathrm{keV}$ maximum energy has been studied. A sawtooth-induced redistribution of more than $50 \%$ of the central fast-ion population has been observed by a novel fast-ion D-alpha (FIDA) diagnostic. The diagnostic uses two arrays of lines of sight and monitors different parts of the fast-ion velocity space. Theoretical predictions by FIDASIM and TRANSP that use the Kadomtsev model to account for the sawtooth-induced fast-ion redistribution are in good agreement with measured spectra and radial profiles. Moreover, the purely co-rotating fast ions are found to be mostly affected by the crashes, while a strong redistribution of fast ions in the broad vicinity of zero pitch is not detected. In contrast to the significant fast-ion redistribution due to the sawtooth crashes, fishbone modes, observed between subsequent sawtooth crashes, do not exhibit a measurable effect on the fast-ion distribution function. This can possibly be explained as fishbone modes only redistribute fast ions in a small region of the phase space.

In dedicated experiments with $5 \mathrm{MW}$ of NBI heating power, the FIDA measurements evidenced a good confinement of fast ions. Radial profiles of the FIDA radiation, measured during on-axis NBI and during off-axis NBI, are in very good agreement with neo-classical predictions from TRNASP. The MHD-induced fast-ion transport, but also a possible fast-ion transport due to turbulence, is consequently below the measurement uncertainties.

In discharges with up to 10 MWof total heating power, the FIDA profiles are slightly flatter than predicted by purely 
collisional transport and the additional fast-ion diffusion coefficient is in the range of $0.5 \mathrm{~m}^{2} / \mathrm{s}$. An investigation of the NBI current-drive efficiency based on data from an MSE diagnostic, a DCN polarimetry diagnostic and the internal inductance agrees with this transport level but also shows that the fast-ion current profiles are modified when varying the NBI injection scheme. A clear broadening of the current profile is measured when replacing on-axis NBI by off-axis NBI.

\section{ACKNOWLEDGEMENTS}

This work has been carried out within the framework of the EUROfusion Consortium and has received funding from the European Unions Horizon 2020 research and innovation programme under grant agreement number 633053. The views and opinions expressed herein do not necessarily reflect those of the European Commission.

[1] STIX, T. H., Plasma Physics 14 (1972) 367.

[2] FISCH, N. J., Rev. Mod. Phys. 59 (1987) 175.

[3] FASOLI, A. et al., Nuclear Fusion 47 (2007) S264.

[4] CHEN, L. et al., Phys. Rev. Lett. 52 (1984) 1122.

[5] MARCUS, F. B. et al., Plasma Physics and Controlled Fusion 33 (1991) 277.

[6] LOVBerG, J. A. et al., Physics of Fluids B: Plasma Physics 1 (1989) 874.

[7] MUSCATEllo, C. M. et al., Plasma Physics and Controlled Fusion 54 (2012) 025006.

[8] NIELSEN, S. K. et al., Plasma Physics and Controlled Fusion 52 (2010) 092001.

[9] KOLESNICHENKO, Y. et al., Nuclear Fusion 40 (2000) 1325.

[10] JONES, O. M. et al., Plasma Physics and Controlled Fusion 55 (2013) 085009.

[11] VON THUN, C. P. et al., Nuclear Fusion 52 (2012) 094010.

[12] ANGIONI, C. et al., Physics of Plasmas (1994-present) 15 (2008) .

[13] HAUFF, T. et al., Phys. Rev. Lett. 102 (2009) 075004.

[14] HEIDBRINK, W. W. et al., Phys. Rev. Lett. 103 (2009) 175001.

[15] REICH, M., 34th EPS Conference on Plasma Phys. Warsaw 31F (2007) P.

[16] GUENTER, S. et al., Nuclear Fusion 47 (2007) 920.

[17] PACE, D. C. et al., Physics of Plasmas 20 (2013) 056108.

[18] GEIGER, B. et al., Plasma Physics and Controlled Fusion 53 (2011) 065010.

[19] GEIGER, B. et al., Nuclear Fusion 54 (2014) 022005.

[20] HEIDBRINK, W. W. et al., Plasma Physics and Controlled Fusion 46 (2004) 1855.

[21] GEIGER, B. et al., Review of Scientific Instruments 84 (2013) .

[22] MLYNEK, A. et al., Review of Scientific Instruments 85 (2014).

[23] PANKIN, A. et al., Computer Physics Communications 159 (2004) 157.

[24] PORCELli, F. et al., Plasma Physics and Controlled Fusion 38 (1996) 2163.

[25] A HERRMANN, O. G. et al., Fusion Science and Technology 44 (2003) 569.

[26] EDITORS, I. P. B. et al., Nuclear Fusion 39 (1999) 2137.

[27] ISLER, R. C., Plasma Physics and Controlled Fusion 36 (1994) 171.

[28] HEIDBRINK, W. et al., Commun. Comput. Phys. 10 (2011) 716.

[29] HEIDBRINK, W. W. et al., Plasma Physics and Controlled Fusion 49 (2007) 1457

[30] SALEWSKI, M. et al., On velocity-space sensitivity of fast-ion D-alpha spectrscopy, submitted 99 (2014) 99.

[31] SALEWSKI, M. et al., Nuclear Fusion 54 (2014) 023005.

[32] RAthGEBER, S. K. et al., Plasma Physics and Controlled Fusion 52 (2010) 095008.

[33] HIRSHMAN, S. P., Physics of Fluids (1958-1988) 21 (1978).

[34] GIANNONE, L. et al., Fusion Engineering and Design 88 (2013) 3299.

[35] LAO, L. et al., Nuclear Fusion 25 (1985) 1421.

[36] CARTHY, P. J. M. et al., Plasma Physics and Controlled Fusion 54 (2012) 015010. 